\title{
The Shapley super-cluster
}

\section{New X-ray detections and mass distribution ${ }^{\star}$}

\author{
E. De Filippis ${ }^{1,2,3}$, S. Schindler ${ }^{4}$, and T. Erben $^{5}$ \\ 1 Dipartimento di Scienze Fisiche, Università degli Studi di Napoli "Federico II", Via Cinthia 9, Compl. Univ. Monte S. Angelo, \\ 80126 Naples, Italy \\ e-mail: betty@na.infn.it \\ 2 MIT Kavli Center for Astrophysics and Space Research, Massachusetts Institute of Technology, 70 Vassar Street, Building 37, \\ Cambridge, MA 02139, USA \\ 3 Astrophysics Research Institute, Liverpool John Moores University, Birkenhead CH41 1LD, UK \\ ${ }^{4}$ Universität Innsbruck, Institut fuer Astrophysik, Technikerstr. 25, 6020 Innsbruck, Austria \\ e-mail: Sabine.Schindler@uibk.ac.at \\ 5 Institut für Astrophysik und Extraterrestrische Forschung (IAEF), Universität Bonn, Auf dem Hügel 71, 53121 Bonn, Germany \\ e-mail: terben@astro.uni-bonn.de
}

Received 21 June 2005 / Accepted 5 August 2005

\begin{abstract}
The largest and the deepest super-structure known today is the Shapley super-cluster. This is the sky area with the highest over-density of galaxy clusters and therefore also an ideal region to test the effects of a high density environment on galaxies and on clusters.

We performed an X-ray survey of a wide region surrounding the Shapley super-structure. Additionally to previously known super-cluster X-ray members, we identified diffuse X-ray emission from 35 cluster candidates without previous X-ray detection. 21 of them were previously known, optically selected super-cluster members, while the other candidates had not been previously detected in any wavelength range. Optical follow-up observations revealed that at least four of these new candidates also have optical cluster counterparts. The super-cluster shows a slightly flattened and elongated morphology. Clusters outside the central dense core are preferentially located in four perpendicular filaments in a similar way to what is seen in simulations of Large Scale Structure.

We measure the cluster number density in the region to be more than one order of magnitude higher than the mean density of rich Abell clusters previously observed at similar Galactic latitudes; this over-density, in the super-cluster outskirts, is mainly due to an excess of low $\mathrm{X}$-ray luminous clusters (with respect to an average population), which leads us to think that the whole region is still accreting low luminosity, small objects from the outskirts. Pushing our total X-ray mass estimate to fainter clusters would drastically increase the total super-cluster mass measure, because of the presence of the rich X-ray low luminosity population.
\end{abstract}

Key words. galaxies: clusters: general - X-rays: galaxies: clusters - cosmology: observations - large-scale structure of Universe

\section{Introduction}

Galaxy clusters are commonly identified as the largest virialized structures in the Universe. Clusters are themselves embedded in larger systems, extending to tens of Mpc; the cores of a few of these super-structures have exceptionally high cluster number densities, and hence are argued to be collapsing under the effect of their own gravity. Detailed measurements of the size, morphology and mass of these collapsing regions are consequently of profound cosmological importance and for a correct understanding of the large-scale structure in the Universe. A considerable observational effort has been devoted in the

$\star$ Table 4 and Appendix A are only available in electronic form at http://www.edpsciences.org past twenty years to the understanding of one of the densest and richest aggregations of galaxy clusters: the Shapley supercluster (SC). This effort has provided a deep knowledge of the internal structure and of the dynamics of the central and brightest clusters and aggregations of clusters in the region.

However, a complete overview and analysis of the whole area in the X-rays is still missing; because of the large angular size only specific regions have been analyzed so far. Our new analysis suggests that previous studies did not include a large portion of the SC clusters because of their high flux limit. Therefore previous determinations of the matter over-density in this region were certainly underestimations and hence also the cosmological conclusions drawn from these analyses suffered from bias. 
The aim of this work is to give a more complete overview of the X-ray properties of extended sources in this exceptionally rich and crowded area of the sky. The paper is organized as follows. Basic information on the Shapley super-cluster are given in Sect. 2. The surveyed sky area is described in Sect. 3. In Sect. 4 we give details of our new algorithm, written to detect extended structures without any a priori model assumption. In Sect. 5 details are given on additional selection criteria applied to our algorithm in order to discriminate non-cluster sources. Lists and tables with the resulting detected clusters and their properties can be found in Sect. 6, together with results on the efficiency of our detection algorithm and a case by case discussion for all clusters undetected in our survey that had previous $\mathrm{X}$-ray detections. In this section we also describe our second step analysis in which we perform a deeper search, to fainter limits, for optically known clusters with no X-ray detection. In Sect. 7 the new cluster candidates detected in our survey and their optical follow-up observations are described. The cluster distribution and the cluster number density in the region, results from the analysis of their X-ray luminosity function and of their cumulative mass profiles, together with optical versus $\mathrm{X}$-ray cluster properties and a discussion on merger rate are given in Sects. 8-10, respectively. Section 11 gives a summary and discussion of the results.

Throughout this paper we quote errors at the $90 \%$ confidence level and, unless otherwise stated, we use $H_{0}=$ $72 \mathrm{~km} \mathrm{~s}^{-1} \mathrm{Mpc}^{-1}\left(\Omega_{\mathrm{m}}=0.3, \Omega_{\Lambda}=0.7\right)$.

\section{The Shapley super-cluster}

By the end of the 1980s a considerable amount of evidence suggested the existence of a small but systematic and significant perturbation on the smooth Hubble flow. Burstein et al. (1986) observed some form of streaming for galaxies within $60 h^{-1} \mathrm{Mpc}$ of the Local Group toward the Centaurus SC in the Southern Hemisphere. It was originally considered a general streaming motion, but then it was believed that these velocities were caused by a Great Attractor in the direction of the Centaurus SC when Scaramella et al. (1989) reported the presence of a very rich concentration of clusters of galaxies, centered at RA $=13^{\mathrm{h}} 05^{\mathrm{m}} 57^{\mathrm{s}} .8$, Dec $=-33^{\circ} 04^{\prime} 03^{\prime \prime} .0$ (J2000). This concentration was estimated to have a distance ranging from $30 h^{-1} \mathrm{Mpc}$ to $200 h^{-1} \mathrm{Mpc}$ with a central peaked component at $145 h^{-1} \mathrm{Mpc}$. About 28 clusters were determined to belong to this concentration in about $2.5 \times 10^{5} h^{-3} \mathrm{Mpc}^{3}$, producing a number over-density of clusters of more than a factor of ten with respect to the mean density of Abell clusters at similar galactic latitudes.

Raychaudhury et al. (1991) reported that the Shapley concentration was not only the most remarkable feature that appears when looking at the spatial distribution of Abell clusters, but that it was also the richest SC in the sky in terms of X-ray emitting clusters.

The discovery of this large concentration of clusters, together with the coherent deviations from the Hubble flow in the direction of Hydra-Centaurus observed in nearby parts of the Universe, led to the hypothesis that the two phenomena could in some way be related.
In the following years many of the cluster galaxies in the central area of the region were observed spectroscopically (Bardelli et al. 1994; Quintana et al. 1995, 1997; Bardelli et al. 1998, 2000). Several of them were also observed in radio wavelengths (Niklas et al. 1995; Venturi et al. 1997; Reid et al. 1998) and many ROSAT pointed observations were devoted to clusters belonging to this superstructure (Bardelli et al. 1996; Schindler 1996; Ettori et al. 1997; David et al. 1999). Some of these objects were observed also with other X-ray satellites such as ASCA (Markevitch \& Vikhlinin 1997; Markevitch et al. 1998; Hanami et al. 1999), BeppoSAX (Ettori et al. 2000; Bonamente et al. 2001; Nevalainen et al. 2001), Einstein (David et al. 1993; Jones \& Forman 1999) and Ginga (Day et al. 1991). Chandra and XMM-Newton were used to observe some of the Shapley clusters (Gastaldello et al. 2003), confirming the strong presence of merging events in the area.

\section{A complete X-ray analysis of the Shapley area}

Originating in the hot intra-cluster gas filling the cluster potential, the X-ray emission provides the means to assess the size, shape and mass of a cluster, offering at the same time a unique opportunity to efficiently detect and to characterize galaxy clusters.

Our aim is to detect and analyze extended sources in a wide region surrounding the Shapley SC. First, we want to detect all known Shapley clusters already confirmed as X-ray emitters. We then aim to verify if we can detect any diffuse X-ray emission from known Shapley clusters that do not yet have any $\mathrm{X}$-ray detection. Finally, we intend to check for the possible presence of unknown extended sources in the area. The application of a uniform detection technique allows a direct comparison of the X-ray properties of all the above sources.

The only possible way to perform a coherent analysis of such a wide area of the sky is to have the whole of it observed with the same instrument and for a reasonably similar amount of time. This kind of data is unfortunately not available from the newest X-ray satellites. The ROSAT All-Sky Survey (RASS) therefore remains a unique archive of data for this type of analysis.

We analyze a region centered on $\mathrm{RA}=13^{\mathrm{h}} 20^{\mathrm{m}} 0.0$, Dec $=$ $-33^{\circ} 00^{\prime} 00^{\prime} 0$ (J2000). The total solid angle covered is $\Omega=$ 0.27 sterad; its size is chosen such that all known cluster members are included. A large border around the SC area is also included in the analysis to allow for a study not only of its central regions, but also of its outskirts.

For this analysis we use the third re-processing of the RASS data, released on March 22nd, 2000 (RASS-III; data can be down-loaded via anonymous ftp from ftp.xray.mpe.mpg.de). 25 plates are analyzed in order to cover the whole selected area. Each field is a superposition of several scans; the exposure time therefore varies from field to field and within each field. A histogram of the exposure time distribution over the whole area is shown in Fig. 1. The mean and median values of the exposure time are $277 \mathrm{~s}$ and $293 \mathrm{~s}$, respectively. 


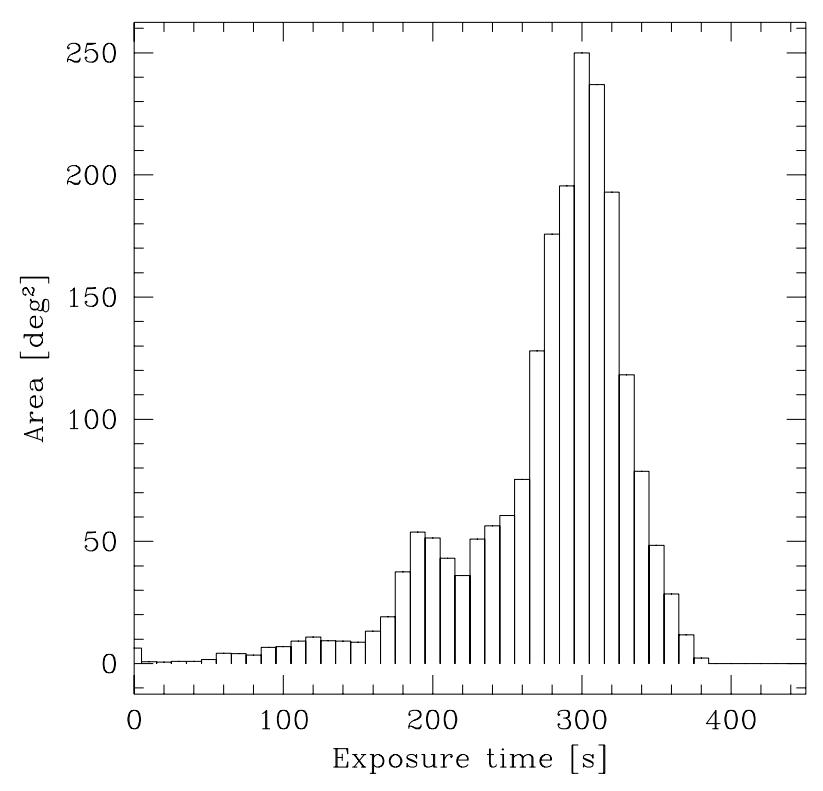

Fig. 1. RASS-III exposure time distribution in the analyzed area.

\subsection{Clusters in the surveyed area}

In the surveyed area there are 768 optically known galaxy clusters. Part of them are grouped to form two large superstructures: the Hydra-Centaurus and the Shapley SCs.

Hydra-Centaurus is located at RA $=13^{\mathrm{h}} 13^{\mathrm{m}} 35^{\mathrm{s}} \cdot 1$, Dec $=$ $-33^{\circ} 21^{\prime} 53^{\prime \prime} 0(\mathrm{~J} 2000.0)$ at a redshift of $z=0.014$. It was believed to be composed by five clusters: Abell 3526, Abell 3537 , Abell 3560, Abell 3565 and Abell 3574 (Einasto et al. 1994), but Abell 3537 and Abell 3560 were later shown to be at higher redshifts. More recently two other clusters were spectroscopically identified as probable SC members: Abell S0753 and Abell 3581.

The Shapley SC lies behind Hydra-Centaurus, almost in the same projected area of the sky, with its center located at RA = $13^{\mathrm{h}} 05^{\mathrm{m}} 57^{\mathrm{s}} .8$, Dec $=-33^{\circ} 04^{\prime} 03^{\prime \prime} .0(\mathrm{~J} 2000.0)$ and $z=0.043$.

174 of the 768 known clusters in the area have no measured redshift. The other 594 are located between $z=0.0114$ (Centaurus clusters) and $z=0.85$ (LCDCS 0831); only five of them are closer than $z=0.03$, while 542 are at $z>0.07$. The remaining 47 clusters are in the intermediate redshift range $0.03 \leq z \leq 0.07$ and hence potential members of the Shapley SC. These we analyze in more detail. We start selecting such a wide redshift range to be sure not to exclude any cluster belonging to the Shapley SC and also (as we did for the sky area to be surveyed) to enable an analysis of the neighboring regions.

The 47 clusters which lie inside the surveyed area and within the selected redshift range are:

- Abell 3558, Abell S0740, Abell 3570, Abell 3575, Abell S0758, Abell 3571, Abell 3578, SC 1336-314, Abell S0757, SC 1329-314, Abell 1736, Abell 3559, Abell 1631, Abell 3554, RX J1332.2-3303, Abell 1644, Abell S0718, Abell 3556, SC 1342-302, Abell 3553, Abell 3555, Abell 3560, Abell S0721, Abell 3562, Abell S0724, Abell 3577, SC 1327-312, Abell S0729, Abell 3544, Abell S0734, Abell S0731, Abell 3564, Abell S0742, Abell 3566,
Abell 3568, Abell 3572, Abell 3552, Abell 1709, Abell 3542, Abell 3537, Abell 3528, RX J1252.5-3116, Abell 3530, Abell 3532, Abell S0726, Abell 3535, Abell S0733.

For a more complete analysis we add three objects which have no measured redshift but which were considered as SC members in previous studies: Abell 3548, Abell 3561 and Abell 3563 (Einasto et al. 1997).

\section{The detection algorithm}

Our choice of the detection algorithm arises from the need to analyze a wide area of the sky and to detect extended and most probably irregular and interacting nearby sources in a high density environment. We therefore need a fast running algorithm which does not make any model assumption on the sources to detect.

Our ad hoc written algorithm directly analyses photon events (which allows a null loss of resolution). Initially a treebased binning of the photon distribution is performed. The aim of the binning is to subdivide the image into the lowest possible number of cells, each containing only one photon. The binning avoids the need to calculate exact distances between all photon pairs in the image; distances between two photons are computed only in a few special cases. This strongly reduces computational time, still allowing an easy, fast and accurate study of the photon positions, keeping the photon event resolution ( 0.5 arcsec) of the initial raw data.

The construction of the tree of cells is performed applying a rectangular division of the two-dimensional space into (sub)cells. The initial image is subdivided into two sub-images, splitting it in the direction perpendicular to its longer side, in such a way that the daughter cells have half the number of particles of the parent image. The two sub-images are then analyzed independently; they are further split perpendicularly to their longer sides, so that their daughter cells have half the number of particles. All daughter cells containing more than one photon are recursively sub-divided into sub-subcells using the above criterion. The binning ends when all subcells contain only one photon.

After subdividing the image into a number of rectangles equal to the number of photons present in the field, the algorithm then searches for spatial density enhancements in the photon distribution with a friends-offriends approach (a modified version of what made publicly available by the University of Washington at: http:// www-hpcc.astro.washington.edu/tools/). This is performed by gathering in the same group all photons which are within a predefined distance $r_{\max }$ of each other. Eventually a group is formed by photons all having at least one "friend" within a distance smaller or equal than the specified $r_{\text {max }}$. Each detected group, formed by at least $N_{\min }$ photons, is considered a candidate cluster. A more detailed description of the algorithm can be found in De Filippis $(2003)^{1}$.

1 A complete version of the Ph.D. thesis can be downloaded from: http ://people.na.infn.it/ betty/tesi_files/PhD.pdf.gz. 


\subsection{Optimizing the algorithm}

In a field containing only sky background a source detection algorithm should detect nothing. We keep this in mind when tuning the two parameters of our algorithm: the search radius $r_{\max }$ and $N_{\min }$, which are the maximum distance within which photons are considered to belong to the same group, and the minimum number of photons a group needs to be considered a candidate cluster, respectively. We hence simulate background photon distributions. In each simulated field we randomly distribute a number of photons equal to the observed average photon number $(\approx 16000)$ in the background of RASS-III fields (each covering $6.4 \times 6.4$ degrees of sky).

When we run our detection algorithm on the simulated background fields, for high values of the search radius $r_{\max }$ and for low values of the minimum number of photons forming each source $-N_{\min ^{-}}$, a high number of sources is detected. On the other hand, if a very small value is assigned to $r_{\max }$ and, at the same time, a group of photons is considered to be a source only if it has a very high number of photons (large values of $N_{\min }$ ), the algorithm retrieves only few sources or, to the extreme, no sources at all. If both the values of $r_{\max }$ and $N_{\min }$ are low, only peaked sources with few photon counts are detected. Larger values of $r_{\max }$ have instead to be chosen if one wants to detect extended sources. For increasing $r_{\max }$, the value of $N_{\min }$ has to increase accordingly, in order to reduce the number of spurious detections.

We choose the couple of values which allows an average detection of only 0.75 sources in each simulated background field:

$$
r_{\max }^{\mathrm{sim}}=230 \mathrm{bins} \quad N_{\min }=17 \text { counts }
$$

where each bin is $0.5^{\prime \prime}$. Real photons are though not randomly distributed because of the large scale structure present in each field, and since the exposure time changes from field to field and is not even constant inside each field.

In order to obtain an unbiased detection, we appropriately weight the values of the search radius $r_{\max }^{\text {sim }}$ chosen from the simulations by the local exposure time and background. To this aim additional data (like event rates, aspect and quality of the data, exposure and background images) are used.

We scale $r_{\max }^{\text {sim }}$ to the true background level within each pixel in each field, taking into account the following simple statistics. Let us consider an area $A$ in which there is a constant density of events $n=N / A$. The probability that an event occurs in a smaller circular area $a=\pi r^{2}$ is equal to $p_{\text {int }}=a / A$; the probability that the event occurs instead outside $a$ is equal to $p_{\text {ext }}=(A-a) / A$. The mean number of events $\langle k\rangle$ inside the region of area $a$ is given by:

$\langle k\rangle=N \cdot p_{\text {int }}=\frac{N}{A} \cdot a=n \cdot a=n \cdot \pi r^{2}$.

Then, the radius inside which $\langle k\rangle$ events are expected to be found can be written, for the simulated fields, as:

$r_{\max }^{\operatorname{sim}}=\sqrt{\frac{\langle k\rangle}{\pi}} \cdot \frac{1}{\sqrt{n_{\text {sim }}}}$ where $n_{\text {sim }}$ is the number of background counts per unit area in the simulations. We then scale $r_{\max }$ proportionally with the local background in the following way:

$r_{\max }(x, y)=\sqrt{\frac{\langle k\rangle}{\pi}} \cdot \frac{1}{\sqrt{n_{\operatorname{RASS}}(x, y)}}$

where $n_{\text {RASS }}$ is the number of local RASS background counts per unit area.

Equations (1) and (2) can be rewritten as:

$$
\begin{aligned}
r_{\max }(x, y)= & r_{\max }^{\operatorname{sim}} \sqrt{\text { bkg. ph. dens. (sim.) }} \\
& \times \frac{1}{\sqrt{\text { bkg. ph. dens. (RASS III) }}(x, y)} .
\end{aligned}
$$

Equation (3) scales the search radius according to the density of the background: in areas with higher background a proportionally smaller $r_{\max }$ will be applied, and vice-versa.

\subsection{Running the algorithm}

On the 25 selected RASS-III plates we run the above described source detection algorithm. Some sky regions are covered by more than one, overlapping, adjacent fields. In such cases the algorithm is run on all available fields; multiple detections are eliminated. Sources located on the border of one of the plates show a mismatch in the measured count-rates within the different plates. In these cases we choose the detection made in the plate where the source is farther away from the border.

$\mathrm{X}$-ray emission coming from the ICM, at temperatures of typically $10^{7}-10^{8} \mathrm{~K}$, occurs predominantly in the hard band. Therefore, in order to obtain the highest possible detection rate with the lowest contamination by non-cluster sources, the cluster detection algorithm is initially run on the photon distribution in the hard ROSAT energy band $(0.5-2.0 \mathrm{keV})$.

For each detected source the following parameters are measured: background-subtracted count rate, number of photons in the hard band and in the soft band, hardness ratio, source center weighted for the varying exposure time inside the source area, total area, radius (mean photon distance from the center of the cluster $-\langle r\rangle)$ and an estimate of the source extent (Ext. $=\langle r\rangle+1.5 \sigma$, where $\sigma$ is the radius containing $68 \%$ of the source photons from the group.)

\section{Cluster detection}

We apply our detection algorithm to the whole surveyed area, detecting a total of 579 sources. A cross-correlation with archival data shows that these can be classified as: 48 galaxy clusters, 90 galaxies, 81 stars, 26 infrared sources (IrS) from the 2 Micron All Sky Survey eXtended sources catalogue (Jarrett et al. 2000), 158 point-like X-ray sources from the ROSAT AllSky Survey Bright Source Catalogue (BSC) (Voges et al. 1999) and 4 galaxy groups. The remaining 172 detected sources are new X-ray detections.

\subsection{Applying further selection criteria}

Finding clusters in X-rays is not an easy task, mainly due to their paucity compared to other types of sources. A large 


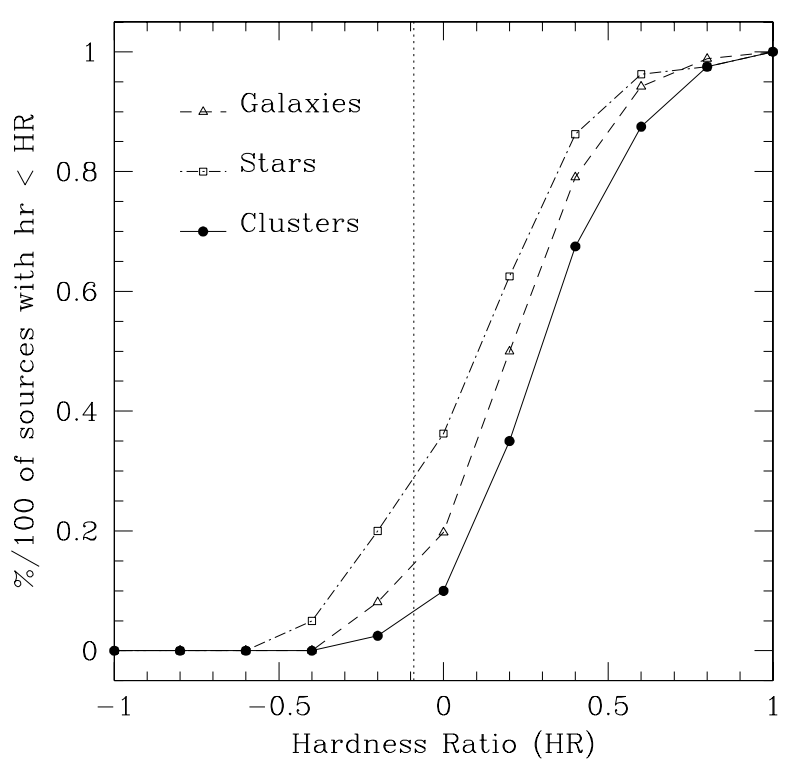

Fig. 2. Cumulative normalized distribution of the hardness ratio of a selection of the known clusters, galaxies and stars among the detected sources.

fraction of the sources detected by applying our detection algorithm to the whole area (see Sect. 5) is in fact given by noncluster sources. Since the main aim of this work is to detect possible new cluster candidates in the area, we apply further selection criteria to all detected sources, with the purpose to exclude the largest possible number of non-cluster sources and spurious detections. We will then be able to perform followup observations only of sources that have a high probability of being cluster candidates.

\subsection{The hardness ratio}

The first selection criterion that we apply is on the spectral hardness of the X-ray emission of each source. Here we use the RASS definition of a source's hardness ratio (HR):

$\mathrm{HR}=\frac{h-s}{h+s}$

where $h$ is the photon count in the hard energy range from 0.5 to $2.0 \mathrm{keV}$ (energy channels 52-201), and $s$ is the photon count in the soft band, from 0.1 to $0.4 \mathrm{keV}$ (energy channels 11-40).

Galaxy clusters have a harder spectrum than other more common X-ray emitting sources. Therefore in principle most clusters are more easily detected in a hard X-ray energy band, where the X-ray background is lower and soft sources are fainter. Unfortunately the ROSAT hard energy band is extremely limited $(0.5-2.0 \mathrm{keV})$, and at a flux of $\approx 10^{-14} \mathrm{erg} \mathrm{s}^{-1} \mathrm{~cm}^{-2}$ clusters still comprise only $10 \%-20 \%$ of the total source population (Hasinger et al. 1993).

In Fig. 2 we show the HR distributions of the subset of our detections, for which optical counterparts were found. The cluster HR distribution (plotted as a solid line) is significantly different from the one for galaxies (dashed line); the distribution for stars (dot-dashed line) lies between the two. A Kolmogorov-Smirnov (KS) test is used to compare the star and the galaxy HR distributions to the cluster one. The probability that the galaxy (star) and the cluster distributions are the same is indeed very low: $P_{\mathrm{KS}}$ (clusters vs. galaxies) $=8.5 \times 10^{-4}$ $\left(P_{\mathrm{KS}}\right.$ (clusters vs. stars) $\left.=8.2 \times 10^{-3}\right)$. There is however no threshold in the HR value which allows a clean discrimination between clusters and other types of sources. The value which provides the best compromise, by allowing us to eliminate the highest number of soft sources while losing the lowest possible number of clusters is:

$\mathrm{HR}=-0.09$

which corresponds to a temperature of $k T \approx 0.5 \mathrm{keV}$.

After applying the above HR selection criterion to all detected sources, we are left with 413 sources among which there are: 40 galaxy clusters, 25 galaxies, 76 stars, 22 infrared sources, 120 point-like X-ray sources and 4 galaxy groups. The remaining 126 sources are new X-ray detections.

\subsection{The source extent}

Clusters of galaxies are much more extended structures than single galaxies or stars. If all sources were at the same distance from us a threshold value in the minimum/maximum extent of a source would be an extremely efficient way to discriminate between extended and point sources. If, however, one is interested in objects at various distances, up to very high redshifts, the source dimension is not such a straightforward selection criterion, and extra care has to be taken in choosing a threshold value. Since we are interested exclusively in low redshift objects, a selection based on the source extent can still be a valid source discriminator.

Figure 3 shows the cumulative distributions of the radial spatial extent (Ext.) for a sub-sample of our detections, for which optical counterparts were found. At low values of Ext., the cluster distribution (plotted as a solid line) is much flatter than the galaxy (dashed line) and the star (dot-dashed line) ones, which show instead a much steeper increase even for low radii. The value which allows the best discrimination between clusters and less extended sources is:

Ext. $=300^{\prime \prime}$,

where the source extent has been defined in Sect. 4.1.

A KS test is applied to the star and the galaxy distributions to compare them with the cluster one. According to this test the two distributions are indeed very different: $P_{\mathrm{KS}}$ (clusters vs. galaxies) $=1.5 \times 10^{-9}$ and $P_{\mathrm{KS}}$ (clusters vs. stars) $=7.8 \times 10^{-13}$.

After applying the above threshold to the source extent of all remaining detected sources we are left with 141 objects. Among these there are 34 galaxy clusters, 25 galaxies, 15 stars, 8 infrared sources, 21 point-like $\mathrm{X}$-ray sources, 4 galaxy groups and 34 previously unknown sources.

\subsection{Minimum count rate}

In order to have a flux lower limit, for all the sources detected in the area, independent of the different exposure times across the field, a count rate threshold of 0.07 counts $\mathrm{s}^{-1}$ in 


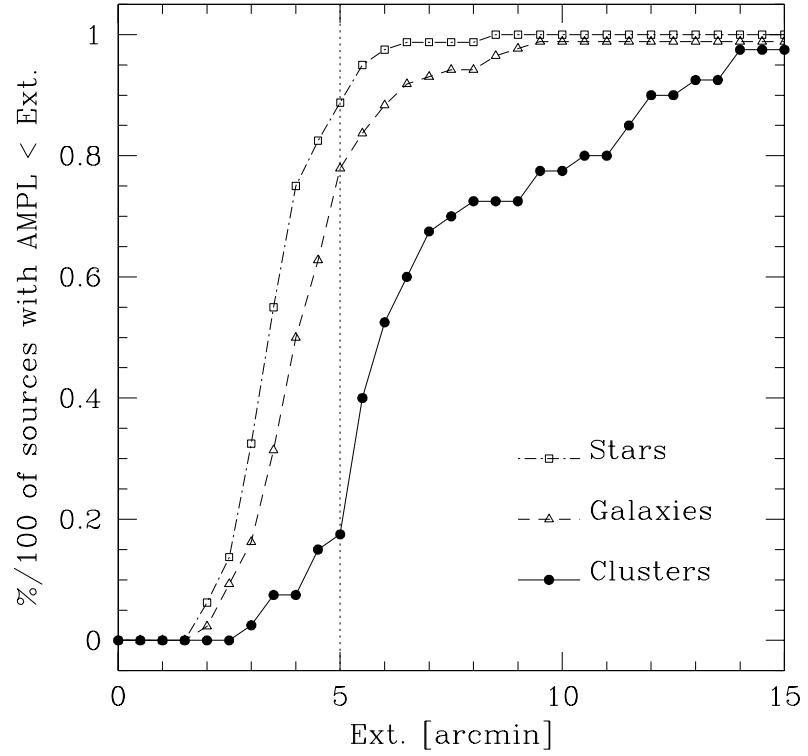

Fig. 3. Cumulative normalized distributions of the spatial extent of a sample of the known stars, galaxies and clusters among all detected sources.

the $0.5-2.0 \mathrm{keV}$ energy band is applied to all the remaining detections; this corresponds approximately to a cut in flux of $f_{\mathrm{X}}(0.1-2.4 \mathrm{keV})=1.55 \times 10^{-12} \mathrm{erg} \mathrm{cm}^{-2} \mathrm{~s}^{-1}$. This last selection leaves a total of $102 \mathrm{X}$-ray detections in the whole area.

\section{Clusters detected in the Shapley region}

After applying our selection criteria to all detected sources, we are left with 102 objects:

- 34 previously known galaxy clusters of which;

- 9 with no previous X-ray detection;

- 23 galaxies;

- 8 stars;

- 7 infrared sources;

- 12 point-like X-ray sources from the BSC;

- 4 galaxy groups;

- 14 new detections: X-ray extended sources for which no counterpart in other wavelengths could be found.

We plan to further investigate the 12 BSC X-ray sources optically, since we detect them as extended structures, and hence can be potential clusters.

In Fig. 4 we plot the percentage of the different types of sources detected which remain after applying our selection criteria: (1) detection algorithm, (2) HR, (3) extent and (4) minimum flux selection criteria were applied. It is clear that we are left with a high percentage of diffuse sources (i.e. clusters and groups of galaxies), as opposed to more point-like and softer sources of which most were rejected. As expected, having applied ad hoc selection criteria to all detected sources has largely improved the performance of the detection algorithm, leading to the rejection of a reasonably low number of clusters (15) and not even one galaxy group, while still performing a good selection against spurious detections and different types of sources.

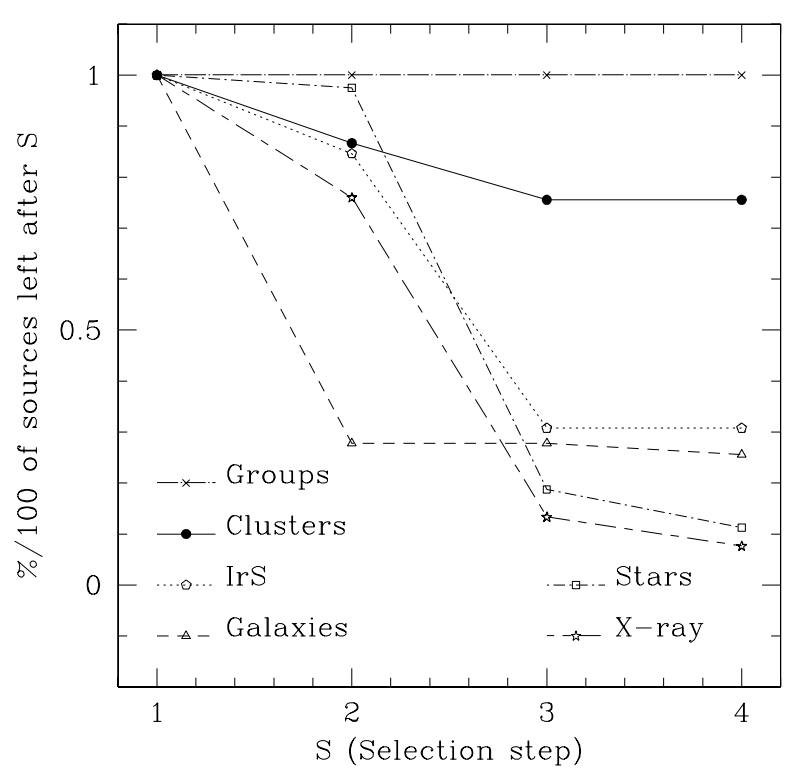

Fig. 4. Percentage of different types of detected sources after each step of our detection and selection process.

Here follows a list of the 34 detected clusters in the area with (1.) and without (2.) previous X-ray detection:

1. Abell S0700, Abell S0701, Centaurus, RX J1252.5-3116, Abell 3528, Abell 3530, Abell 1644, Abell 3532, Abell 1648, Abell 3541, Abell 3548, Abell 1732, Abell 1736, Abell 3558, SC 1327-312, SC 1329-314, RX J1332.2-3303, Abell 3562, Abell 1757, Abell 3563, Abell 3565, Abell 3570, Abell 3571, Abell 3574, Abell 3581.

2. Abell S0717, Abell S0721, Abell S0753, Abell 1604, Abell 1633, Abell 1791, Abell 3519, Abell 3535, Abell 3557.

Seventeen of these clusters are in the selected redshift range $(0.03 \leq z \leq 0.07$, see Sect. 3.1); unless otherwise stated, from now on we will limit our analysis to clusters within this redshift range. In part of our following analysis we will also consider the 14 new X-ray detections, which also are hard, extended sources located in the area, and are therefore probable SC members.

Eight clusters inside the selected redshift range, with known optical counterparts, are identified by the detection algorithm but excluded during one of the three selection steps. These clusters are:

- Abell S0758, Abell 1631, Abell 3554, Abell S0718, Abell 3556, Abell 3553, Abell S0724, Abell S0729.

Four of them are excluded because of their relatively low extent; the remaining four clusters have instead extremely low values of the HR. These sources are known to be clusters, because of their optical counterparts, and are all above our flux limit; we therefore include them in our following analysis of the SC since they were detected with the same technique as for all other clusters. 


\subsection{Cluster properties}

\subsubsection{Average temperature}

For all detected objects we estimate the average gas temperature applying the following method by Ebeling et al. (1996).

1. The flux in the energy band $0.1-2.4 \mathrm{keV}$ is initially measured assuming a cluster temperature of $5.0 \mathrm{keV}$;

2. The corresponding luminosity is computed;

3. The temperature is derived from the X-ray luminosity through (Markevitch et al. 1998):

$$
k T=6[\mathrm{keV}]\left(\frac{L_{\mathrm{X}}\left[10^{44} \mathrm{erg} \mathrm{s}^{-1}\right]}{1.41 h^{-2}}\right)^{\left(\frac{1}{2.10}\right)}
$$

4. The estimated temperature is used to recompute the flux.

Steps from (2) to (4) are repeated iteratively until the results converge, which most often happens after no more than three iterations.

Table 4 summarizes measured and estimated quantities for detected clusters and for the new X-ray detections. For some of these clusters (Abell 3535, Abell S0718, Abell S0721, Abell S0724, Abell 3553, Abell 3554, Abell S0729, Abell S0758), and naturally for all the new detections, this is their first X-ray detection; values in Table 4 are therefore today the only X-ray data available for these clusters. When available the literature, we quote average gas temperatures obtained from spectral analysis; the respective references are also listed. We observe a good agreement between our temperature estimates and values measured in literature.

\subsubsection{X-ray versus optical position}

In Fig. 5 we compare the optical coordinates of all detected clusters with the center of our X-ray detection. We observe a general agreement between their centers in the two wavebands with no systematic deviation. The few exceptions are represented by extraordinarily extended clusters or clusters showing significant sub-structures in their X-ray emission. Even if the peak in the optical luminosity does not always coincide with the X-ray one, it falls anyway within the X-ray extent of the cluster.

\subsection{Detection efficiency}

The main aim of this work is to detect the largest possible number of extended structures that lie in the Shapley SC. We stress that we do not aim to build a complete sample, but mainly to detect new possible Shapley members in order to provide a more reliable mass estimate and morphological description of the whole super-structure. In this section we briefly discuss the detection efficiency of our algorithm by considering the performance on known galaxy-clusters at low redshift. Within the surveyed area there are 52 optically known galaxy clusters with redshift $z \leq 0.07$, of which 47 belong to the Shapley SC. To this sample we add in our studies three more candidates with no redshift estimate which have been considered as SC members
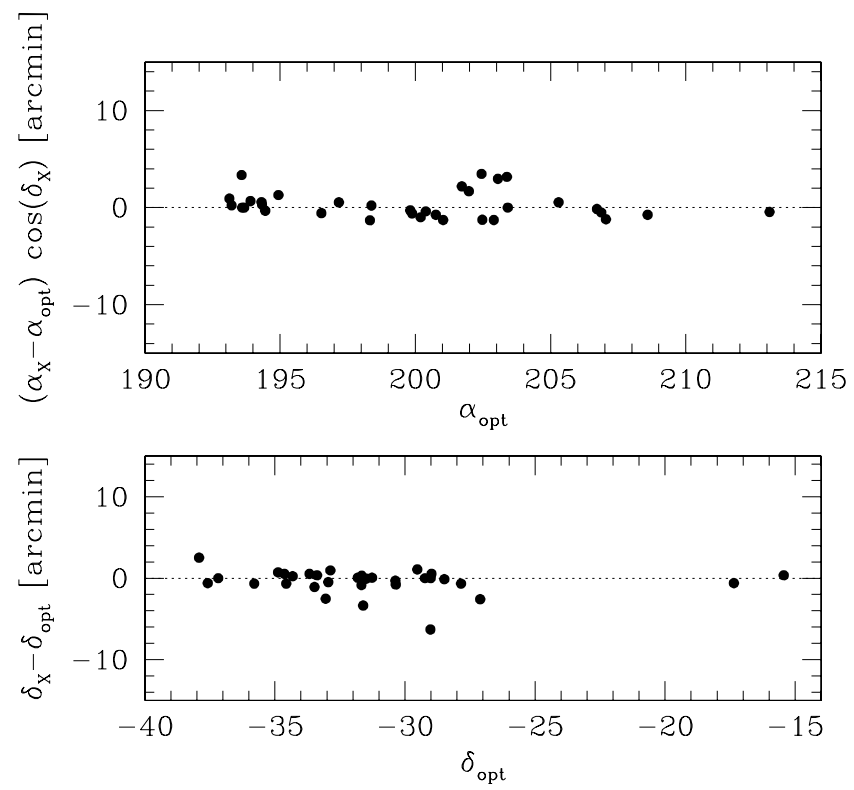

Fig. 5. Comparisons, for the detected clusters, of their optical coordinates with their X-ray detection coordinates. RA and Dec are separately analyzed to check for systematic deviations.

in the past (Sect. 3.1). We detect $56 \%$ (30) of these 55 clusters applying our algorithm, as outlined in Sect. 5. While we miss a significant fraction of real clusters with our conservative selection criteria, we substantially reduce the contamination of our sample with non-cluster sources. In our final cata$\log$ of 102 objects we have 38 known galaxy groups/clusters, 50 point sources and 14 new X-ray detections. Regarding only the known sources we can expect that about $40 \%$ of the new detections are hitherto unknown galaxy groups/clusters.

For each optically known cluster which we do not detect with the blind run of our algorithm we perform a refined second step analysis (see Sect. 6.3).

\subsection{Second step analysis}

Among the optically known galaxy clusters in the area, within the selected redshift range, there are 25 clusters which were not detected by our algorithm. Only four of these 25 clusters (Abell 1709, Abell 3559, Abell 3560 and Abell 3566) have previous X-ray detections. Their cases are discussed in detail in the following sections.

For these 25 sources we perform a further analysis to verify if any X-ray emission is present at their location. We run our detection algorithm lowering the minimum number of photons allowed in a group, and applying no further cuts. Despite the low threshold we can not detect any X-ray emission for 10 of the 25 clusters:

- Abell 3544, Abell 3555, Abell S0731, Abell S0733, Abell 3559, Abell 3560, Abell 3561, SC 1342-302, Abell S0740, Abell 3578.

For the remaining 15 clusters:

- Abell 3537, Abell 3542, Abell S0726, Abell 1709, Abell 3552, Abell S0734, Abell 3564, SC 1336-314, Abell 3566, 
Table 1. Sources detected in the second step analysis.

\begin{tabular}{|c|c|c|c|c|c|c|c|c|c|c|}
\hline Cluster & $\begin{array}{c}\text { RA; Dec } \\
(\mathrm{J} 2000.0) \\
(\mathrm{deg})\end{array}$ & $z$ & $\begin{array}{c}n_{\mathrm{H}} \\
\left(10^{20}\right. \\
\left.\mathrm{cm}^{-2}\right)\end{array}$ & $\begin{array}{l}\text { Radius } \\
\text { (arcmin) }\end{array}$ & HR & $\begin{array}{l}\text { Count Rate } \\
\left(\text { counts s }^{-1}\right)\end{array}$ & \begin{tabular}{r}
\multicolumn{1}{c}{$f_{\mathrm{X}}$} \\
$\mathrm{erg} \mathrm{cn}$
\end{tabular} & $\begin{array}{l}\frac{f_{\text {bol }}}{-12} \\
\left.-2 \mathrm{~s}^{-1}\right)\end{array}$ & $\begin{array}{c}L_{\mathrm{bol}} \\
\left(10^{44}\right. \\
\left.\mathrm{erg} \mathrm{s}^{-1}\right)\end{array}$ & $\begin{array}{c}k T \\
(\mathrm{keV})\end{array}$ \\
\hline Abell 3537 & $195.255 ;-32.423$ & 0.0320 & 5.70 & 2.80 & +0.47 & 0.034 & 0.85 & 1.13 & 0.070 & 0.92 \\
\hline Abell 3542 & $197.201 ;-34.489$ & 0.0525 & 4.82 & 1.82 & +0.00 & 0.017 & 0.46 & 0.65 & 0.040 & 0.69 \\
\hline Abell S0726 & $198.806 ;-33.695$ & 0.0590 & 4.78 & 2.79 & +0.43 & 0.026 & 0.55 & 0.85 & 0.069 & 0.90 \\
\hline Abell 1709 & $199.690 ;-21.488$ & 0.0521 & 8.01 & 5.16 & +0.22 & 0.052 & 1.30 & 1.73 & 0.106 & 1.12 \\
\hline Abell 3552 & $199.753 ;-31.851$ & 0.0520 & 4.72 & 1.48 & +0.20 & 0.018 & 0.49 & 0.70 & 0.041 & 0.70 \\
\hline Abell S0734 & $202.006 ;-41.106$ & 0.0503 & 6.75 & 1.72 & +0.67 & 0.017 & 0.51 & 0.71 & 0.040 & 0.67 \\
\hline Abell 3564 & $203.660 ;-35.228$ & 0.0505 & 4.08 & 1.14 & +0.43 & 0.016 & 0.46 & 0.62 & 0.035 & 0.66 \\
\hline SC 1336-314 & $204.071 ;-31.788$ & 0.0395 & 3.82 & 2.47 & +0.20 & 0.025 & 0.72 & 0.98 & 0.034 & 0.64 \\
\hline Abell 3566 & $204.762 ;-35.610$ & 0.0510 & 4.16 & 1.04 & +0.75 & 0.021 & 0.56 & 0.76 & 0.044 & 0.72 \\
\hline Abell 3568 & $205.328 ;-34.652$ & 0.0516 & 4.10 & 1.67 & +0.43 & 0.016 & 0.46 & 0.62 & 0.036 & 0.66 \\
\hline Abell S0742 & $206.107 ;-34.305$ & 0.0510 & 4.41 & 1.57 & +0.71 & 0.018 & 0.50 & 0.69 & 0.040 & 0.68 \\
\hline Abell 3572 & $207.100 ;-33.394$ & 0.0517 & 4.13 & 2.22 & +0.39 & 0.026 & 0.66 & 0.89 & 0.052 & 0.79 \\
\hline Abell 3575 & $208.170 ;-32.920$ & 0.0377 & 4.50 & 2.42 & +0.25 & 0.030 & 0.85 & 1.17 & 0.037 & 0.66 \\
\hline Abell 3577 & $208.574 ;-27.895$ & 0.0494 & 4.67 & 1.29 & +1.00 & 0.020 & 0.55 & 0.75 & 0.041 & 0.70 \\
\hline Abell S0757 & $213.035 ;-33.086$ & 0.0440 & 4.76 & 2.17 & -0.20 & 0.018 & 0.55 & 0.74 & 0.032 & 0.63 \\
\hline
\end{tabular}

Column (1): source name; Col. (2): RA and Dec(J2000); Col. (3): redshift; Col. (4): galactic HI column density in units of $\left(10^{20} \mathrm{~cm}^{-2}\right)($ Dickey \& Lockman 1990); Col. (5): source radius (arcmin); Col. (6): hardness ratio; Col. (7): count rate in the 0.5-2.0 keV energy band; Cols. (8) and (9): $f_{\mathrm{X}}$ in the $0.1-2.4 \mathrm{keV}$ energy band and bolometric flux both in units of $\left(10^{-12} \mathrm{erg} \mathrm{cm}^{-2} \mathrm{~s}^{-1}\right)$; Col. (10): bolometric luminosity in units of $\left(10^{44} \mathrm{erg} \mathrm{s}^{-1}\right)$; Col. (11): cluster average temperature in $\mathrm{keV}$.

Table 2. Optically known and detected clusters.

\begin{tabular}{llcccc}
\hline \hline & & $z<0.03$ & $0.03 \leq z \leq 0.07$ & $0.0446 \leq z \leq 0.0554$ & $\begin{array}{c}0.0446 \leq z \leq 0.0554 \\
208.74 \leq \mathrm{RA} \leq 193.14 \\
-37.64 \leq \text { Dec } \leq-27.15\end{array}$ \\
\hline 1 & $N_{\mathrm{cl}}$ & 5 & $46(+1)$ & $34(+1)$ & $30(+1)$ \\
\hline 2 & Detected & 5 & 17 & 14 & 11 \\
3 & Detected but excl. & - & 8 & 7 & 6 \\
4 & Det. in 2nd step & - & 15 & 10 & 8 \\
\hline 5 & TOT detected & 5 & 40 & 31 & 25 \\
\hline
\end{tabular}

Column (1): line number; Col. (2): table legend. Number of clusters optically known (line 1), detected by our algorithm (line 2), identified by the detection algorithm but excluded during one of the three selection steps (line 3), detected during the second step analysis (line 4); in line 5 is the total number of clusters detected in this work (=line $2+3+4)$. The number of clusters are listed in different redshift ranges; Col. $(3)$ : $z<0.03$; Col. (4): $0.03 \leq z \leq 0.07$; Col. (5): within the redshift range of the core of the Shapley SC (0.0446 $\leq z \leq 0.0554$ ); Col. (6): in the sky region of the core of the Shapley SC. In line 1 we list RX J1332.2-330 in parenthesis since from our analysis shows up as a probable coincidence with Abell 3560 (see Sect. 6.3.3).

Abell 3568, Abell S0742, Abell 3572, Abell 3575, Abell 3577, Abell S0757

we instead detect signs of faint X-ray emission. Properties of these second step detections are listed in Table 1, while in Table 2 we provide the number of optically known and detected clusters located in the redshift ranges and sky regions analyzed in this paper.

\subsubsection{Abell 1709}

The only previous X-ray detection of Abell 1709 is by Jones \& Forman (1999) from pointed observations with the Einstein satellite. Within a radius of $1 \mathrm{Mpc}$ they measure a cluster $\mathrm{X}$-ray luminosity of $L_{\mathrm{X}}(0.5-4.5 \mathrm{keV})=0.176 \times 10^{44} \mathrm{erg} \mathrm{s}^{-1}$ (transformed for our assumed cosmology); this corresponds to a bolometric luminosity of $L_{X}(\mathrm{bol})=0.296 \times 10^{44} \mathrm{erg} \mathrm{s}^{-1}$ and to a flux of $f_{\mathrm{X}}(0.1-2.4 \mathrm{keV})=3.63 \times 10^{-12} \mathrm{erg} \mathrm{cm}^{2} \mathrm{~s}^{-1}$. At the local exposure time of $t_{\exp }=256 \mathrm{~s}$ the cluster should show a total of 37 photons. We instead detect only 15 photons for this source. Because of the high background and the faint signal from the source, we can only trace the cluster out to a radius of $0.3 \mathrm{Mpc}$; this might partially cause the observed flux discrepancy.

\subsubsection{Abell 3559}

The only previous X-ray detection of Abell 3559 is by David et al. (1999) from pointed ROSAT observations. Within a radius of $1 \mathrm{Mpc}$ they estimate a bolometric luminosity for the 


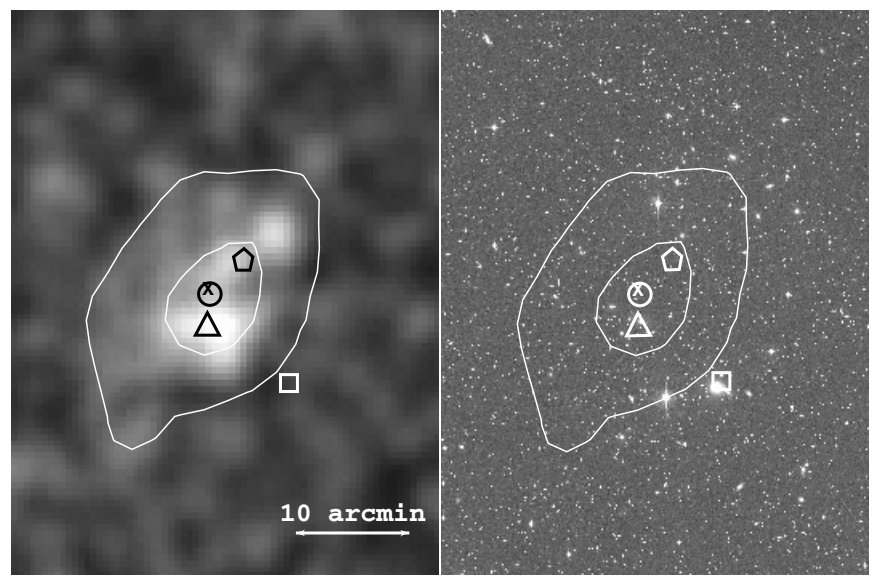

Fig. 6. Abell 3560. Left: smoothed X-ray image with superimposed $\mathrm{X}$-ray contours at 0.2 and 0.4 counts $\mathrm{px}^{-1}$. The empty square and pentagon point to the positions of Abell 3560 and RXJ 1332.2-3303; the cross is for the location of the detection of RXJ 1332.2-3303 in this work and the circle and triangle point to the locations of the detections of Abell 3560 by Ebeling et al. (1996) and David et al. (1999). Right: optical DSS image.

cluster of $L_{\mathrm{X}}(\mathrm{bol})=0.226 \times 10^{44} \mathrm{erg} \mathrm{s}^{-1}$, which corresponds to a flux of $f_{\mathrm{X}}(0.1-2.4 \mathrm{keV})=3.4 \times 10^{-12} \mathrm{erg} \mathrm{cm}^{-2} \mathrm{~s}^{-1}$. At the local exposure time of $t_{\exp }=294 \mathrm{~s}$ this would correspond to a total of 42 cluster photons. This is much higher than our flux limit and hence we would expect to detect this source, but neither the hard nor the soft RASS-III images show any increase above the background emission at the cluster position. Deeper $\mathrm{X}$-ray observations of this object are needed to clarify this discrepancy.

\subsubsection{Abell 3560}

Abell 3560 was already detected in X-rays by Ebeling et al. (1996) using RASS-I data and by Ettori et al. (1997) and by David et al. (1999) through ROSAT pointed observations. Their values of the cluster bolometric luminosities, converted to our cosmology, are: $L_{X}(\mathrm{bol})=1.18 \times 10^{44} \mathrm{erg} \mathrm{s}^{-1}, L_{X}(\mathrm{bol})=$ $1.16 \times 10^{44} \mathrm{erg} \mathrm{s}^{-1}$ and $L_{X}($ bol $)=1.40 \times 10^{44} \mathrm{erg} \mathrm{s}^{-1}$, respectively.

In the left panel of Fig. 6 is the RASS hard X-ray image of A 3560. A bright, extended and irregular source is observed close to the position of RX J1332.2-3303 (pentagon), while there is no detectable X-ray emission at the location of Abell 3560 (empty box). The centers of the X-ray detections of both Ebeling et al. (1996) and David et al. (1999) (plotted as an empty circle and triangle, respectively) lie close to the position in the sky of RX J1332.2-3303 and well within the extent of our X-ray detection; the distance to Abell 3560 is instead much larger $\left[d_{\mathrm{RX}}=4.2^{\prime}\right.$ and $d_{\mathrm{Ab}}=11.5^{\prime}$ for Ebeling et al. (1996) and $d_{\mathrm{RX}}=6.5^{\prime}$ and $d_{\mathrm{Ab}}=10.2^{\prime}$ for David et al. (1999)]. Our X-ray detection of RX J1332.2-330 (cross) is coincident with the detection of Abell 3560 by Ebeling et al. (1996). Ettori et al. (1997) do not explicitly give coordinates for their detection; no comparison with their work was therefore possible.
We are therefore inclined to think that either the X-ray emission of Abell 3560 is too faint to be observable even in pointed observations, or more probably that Abell 3560 and RX J1332.2-3303 are the same cluster and that the coordinate mismatch is simply due to the fact that the optical position was centered on a bright galaxy which lies on the outer edges of the cluster.

Assuming the latter hypothesis to be true, we recompute our bolometric luminosity for RX J1332.2-3303 using the value of the temperature $(k T=3.4 \mathrm{keV})$ measured by David et al. (1999) and the redshift of Abell 3560; we obtain a value $L_{\mathrm{X}}(\mathrm{bol})=1.13 \times 10^{44} \mathrm{erg} \mathrm{s}^{-1}$ which is only slightly lower than those computed by Ebeling et al. (1996), Ettori et al. (1997) and David et al. (1999); we see the same trend in our estimate of the cluster temperature (see Table 4).

\subsubsection{Abell 3566}

As for Abell 3559, also for Abell 3566 one previous detection in X-rays is present in the literature (by David et al. (1999) using ROSAT pointed observations). Within $1 \mathrm{Mpc}$ they compute a cluster luminosity of $L_{X}(0.5-2.0 \mathrm{keV})=$ $0.029 \times 10^{44} \mathrm{erg} \mathrm{s}^{-1}$; this corresponds to a bolometric luminosity of $L_{X}(\mathrm{bol})=0.086 \times 10^{44} \mathrm{erg} \mathrm{s}^{-1}$ and to a flux of $f_{\mathrm{X}}(0.1-2.4 \mathrm{keV})=1.10 \times 10^{-12} \mathrm{erg} \mathrm{cm}^{-2} \mathrm{~s}^{-1}$, which is below our lower flux limit. Furthermore, in the hard band, where we initially detect the sources, the cluster would have a count rate of: $\operatorname{cr}(0.5-2.0 \mathrm{keV})=4.2 \times 10^{-2}$ counts s$^{-1}$, which at the local exposure time of $t_{\exp }=323 \mathrm{~s}$ gives a total number of photon counts for the whole source of $N_{\text {tot }}=13$, which is below the minimum limit fixed in the algorithm (see Sect. 4.1). We therefore detect this cluster only in the second step of our analysis. From the RASS data the cluster is barely detectable and appears almost point-like, with a radius of $\sim 100 \mathrm{kpc}$. We therefore detect only a small percentage of its emission, which causes an even lower detected flux of: $f_{\mathrm{X}}(0.1-2.4 \mathrm{keV})=$ $0.56 \times 10^{-12} \mathrm{erg} \mathrm{cm}^{-2} \mathrm{~s}^{-1}$.

\section{New X-ray detections}

Besides already known groups and clusters of galaxies, we also detect 14 new cluster candidates, which also are hard, extended X-ray sources above our flux limit, but have no previous $\mathrm{X}$-ray detection and no known optical counterpart. Details of the X-ray properties of these 14 sources (labeled as B1-B14) are given in Table 4.

To verify these cluster candidates in the optical we have performed follow-up observations.

\subsection{Optical follow-up}

The primary aim of this follow-up program is the identification of optical counter parts for the X-ray peaks. In particular we want to closer investigate whether these sources are hitherto unidentified members of the Shapley SC. To this end we try to identify galaxy over-densities and to look for Red Cluster sequences from elliptical cluster galaxies at $z \approx 0.05$. 
We obtained optical imaging data for 11 out of the 14 fields $^{2}$ with WFI@ESO2.2m having a field-of-view of $34^{\prime} 0 \times 34^{\prime} 0$ (ESO Observing Programs 71.A-0430(A,B); P.I. S. Schindler). For each candidate we acquired $2350 \mathrm{~s}$ (divided in 5 exposures a $470 \mathrm{~s}$ ) in broad-band $R$ and $2010 \mathrm{~s}$ (divided in 3 exposures a $670 \mathrm{~s}$ ) in broad-band $B$. The fields were observed by ESO in queue scheduling from April 2003 to September 2003 mostly under non-favorable photometric conditions. The processing of the data was done with the image processing pipeline and the methods described in Schirmer et al. (2003); Erben et al. (2005) and the details are not repeated here. The seeing in the final stacked mosaic images ranges from 1.0 to 1.8 arcsec and because of the lack of usable standard star observations we estimated approximate magnitude zero-points by matching the galaxy number counts to WFI observations of the Chandra Deep Field South (Giavalisco et al. 2004).

We give a detailed discussion of our findings for each candidate in App. A. Our main result is that we consider the cases B1, B4, B5 and B6 as good candidates for new galaxy clusters. In these cases, we see a clear light over-density from galaxies close to the X-ray emission peaks and have indications for a Red Cluster sequence in the color-magnitude plots. In the course of our work we noticed that our candidate B5 corresponds to the Abell cluster 3538. Only further observations (e.g. spectroscopy) can finally clarify the nature of these sources. If they indeed are members of the Shapley SC they all represent, as expected, optically poor galaxy cluster systems (Sect. 9). For the cases B7, B9, B11, B12 and B13 the most probable explanation for the X-ray emission at this stage are point sources and we do not think that the X-ray flux in these fields originates from Shapley SC members. No conclusive results are obtained in the cases B8 and B14. In B8, probably most of the $\mathrm{X}$-ray emission comes from a galaxy group at $z \approx 0.01$ but we also see a slight light over-density at the $\mathrm{X}$-ray peak position. This candidate is included in the REFLEX galaxy cluster catalog (Böhringer et al. 2004). B14 is located in a dense stellar field but shows an extended light over-density at the X-ray peak.

\section{Cluster number density}

Figure 7 shows a histogram of the number of clusters in the region, within the $0.03 \leq z \leq 0.07$ redshift range, as a function of their co-moving separation from Abell 3558; we choose this cluster as a reference point since it is one of the most luminous and therefore most massive clusters in the whole area and it is located at the center of the densest region of the super-cluster. The co-moving distance between two clusters is defined as:

$R_{\mathrm{A} 3558, \mathrm{i}}=\sqrt{D_{\mathrm{A} 3558}^{2}+D_{\mathrm{i}}^{2}-2 D_{\mathrm{A} 3558} D_{i} \cos \phi}$

where $D_{\mathrm{A} 3558}$ and $D_{i}$ are the co-moving distances to Abell 3558 and to the cluster $i$, respectively, while $\phi$ is the angular separation between Abell 3558 and the cluster $i$. Over-plotted with a bold line is the histogram of the clusters which have been detected in this work.

\footnotetext{
${ }^{2}$ No observations or data of very bad quality were obtained for fields B2, B3 and B10.
}

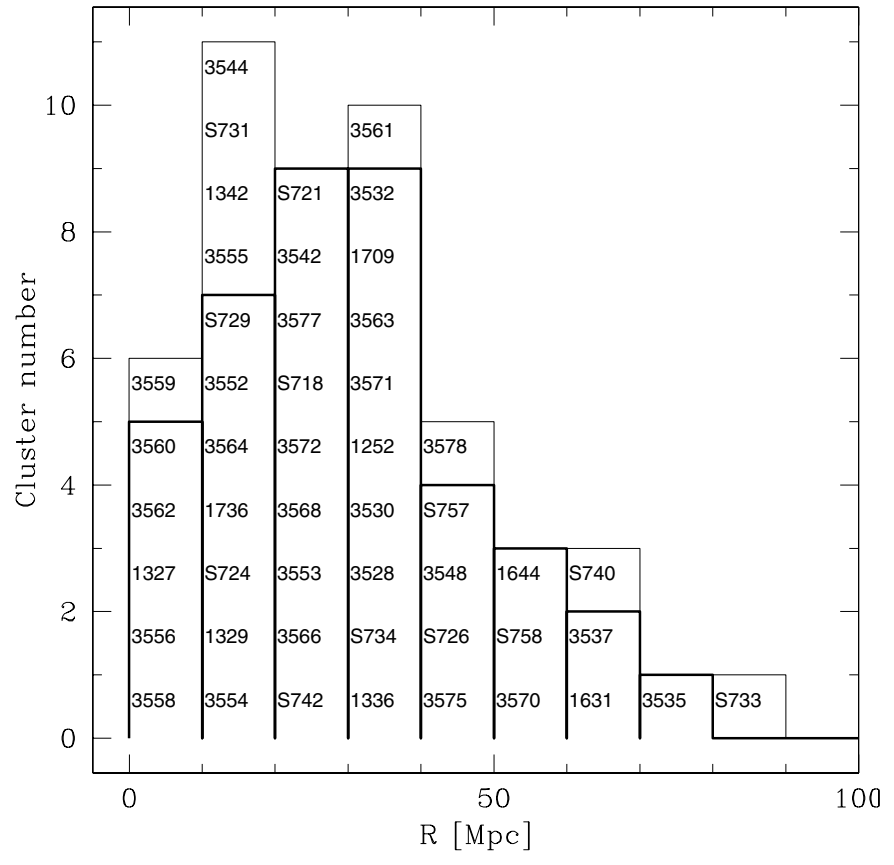

Fig. 7. Histogram of the number of clusters as a function of their comoving distance from the cluster Abell 3558. Over-plotted with a bold line is the histogram of the clusters detected in this work.
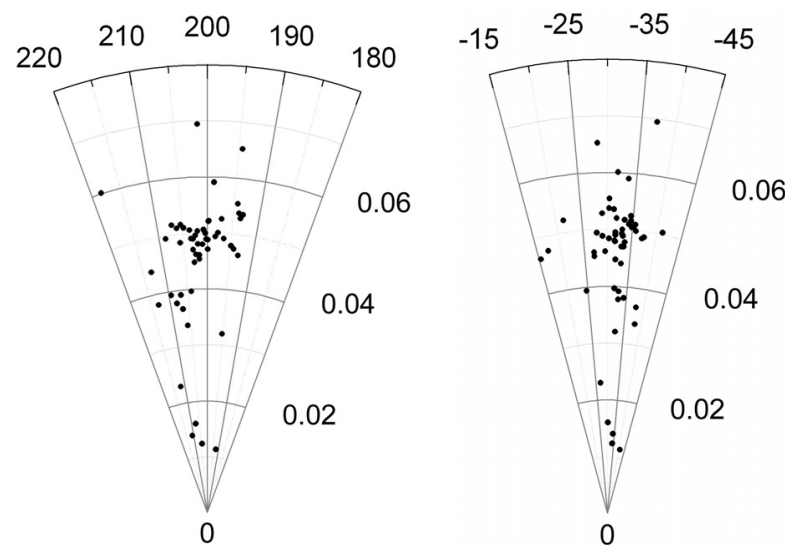

Fig. 8. Left and right panels: Right Ascension and Declination versus redshift for all the clusters inside the selected redshift range.

Two peaks in the cluster distribution can be seen; they are due to the presence of two dense cluster concentrations. The first, more massive one, is centered at RA $=202.35$, Dec $=-31.88$ and lies around Abell 3558; the second, smaller concentration, is centered at RA $=194.26$, Dec $=-30.04$ and is at a distance of $\approx 35 \mathrm{Mpc}$ from Abell 3558 .

The two panels in Fig. 8 show the distribution of all known clusters in the selected area, which have a measured redshift lower than 0.08 . The Shapley SC clearly appears as a dense and compact concentration of clusters with the densest core lying within redshifts $z=0.044$ to $z=0.055$. A smaller aggregate of 8 clusters lies in the foreground at slightly lower redshifts $(0.04<z<0.03)$, while just a few clusters lie randomly dispersed in the background.

Figure 9 shows the positions of all known clusters in the area. The high cluster density strip is caused by the 


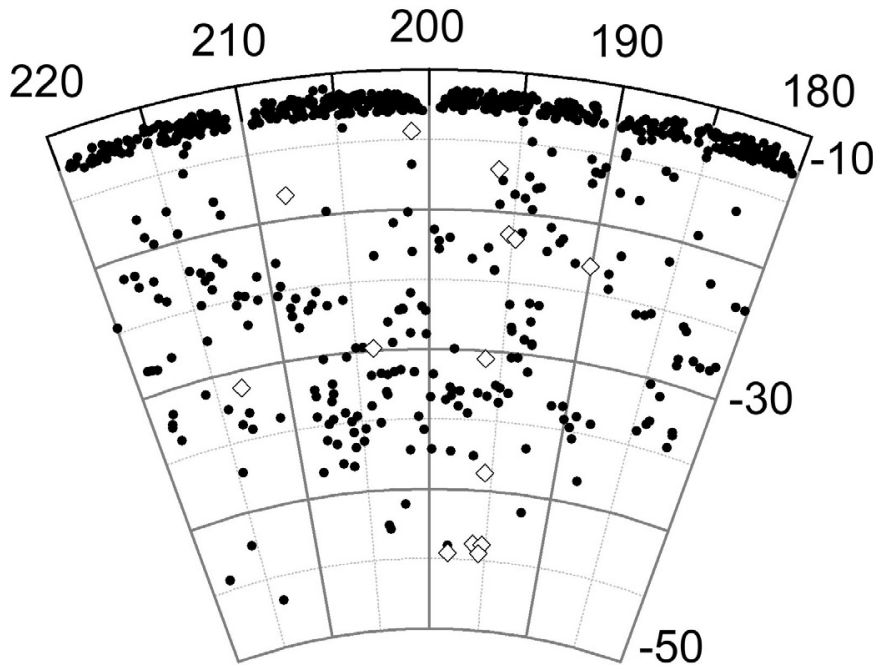

Fig. 9. Positions of all known clusters in the area. Empty squares indicate the new detections from this work while filled circles give the positions of all other clusters.

Las Campanas Distant Cluster Survey (Gonzalez et al. 2001) which scanned a strip along the Right Ascension $85^{\circ}$ long and with a narrow width in declination $\left(1.5^{\circ}\right)$. Figure 10 shows the positions of all known clusters inside the selected redshift range. In the top panel is a plot of their RA versus their Dec In the bottom panel the clusters are plotted in a three-dimensional "orthogonal projection".

Inside the selected redshift range we detect a total of 54 extended sources, spread over a total volume of $\approx 1.42 \times 10^{6} \mathrm{Mpc}^{3}$. Their distribution is centered at RA $=201.14^{\circ}, \mathrm{Dec}=-32.43^{\circ}$ and has a mean redshift of $z=0.048$. The projection of the cluster positions on the three orthogonal planes in the bottom panel of Fig. 10 shows that the superstructure is overall slightly flattened and elongated; at the center of the super-structure we can see a dense core, while clusters located outside this core are positioned along four perpendicular filaments (see projection on plane $y=0$ ), in a strikingly similar way to what seen in simulations of Large Scale Structure (Jenkins et al. 1998; Yoshida et al. 2001). The SC core seems therefore to be created at the intersection of almost perpendicular filaments and it is probably still accreting further clusters through this surrounding filamentary large scale structure. It is further interesting to notice that also all our new candidate clusters either lie in the SC core or along the surrounding filaments. The central dense core of the SC is located between: $193.14 \leq \mathrm{RA} \leq$ $208.74,-37.64 \leq$ Dec $\leq-27.15$ and $0.0446 \leq z \leq 0.0554$. In it we detect (in a volume of $7.43 \times 10^{4} \mathrm{Mpc}^{3}$ ) 25 optically known clusters. This leads to a cluster number density of $\approx 3.4 \times 10^{-4} \mathrm{Mpc}^{-3}$ representing a number density of two orders of magnitude higher than the mean density of rich Abell clusters $\left(\rho_{\text {Abell }} \approx 3.4 \times 10^{-6} \mathrm{Mpc}^{-3}\right)$ observed at similar Galactic latitudes (Abell et al. 1989). Our estimate is about one order of magnitude higher than previous optical measures of the cluster number density of the Shapley $\left(\rho_{\text {Shapley }} \approx 3.4 \times 10^{-5} \mathrm{Mpc}^{-3}\right.$, Scaramella et al. 1989). If our new cluster candidates are also included, the cluster number density will even further increase.

\section{The X-ray luminosity function}

A direct measure of the cluster abundance and population in the Universe is the X-ray luminosity function (XLF), which is defined as the volume density of clusters per luminosity interval. Comparisons of the observed cluster abundance distribution as a function of their luminosity and/or mass with specific cosmological models are fundamental tests for theoretical models and statistics of large scale structure. Recently the availability of large cluster samples has made it possible to reduce the statistical scatter and tightly constrain the XLF (Ebeling et al. 2000; Böhringer et al. 2001; Mullis et al. 2004). The strong correlation between the X-ray luminosity of a cluster and its total mass, makes it then possible to transform the XLF into a mass function also for large samples of clusters.

We compute the XLF both for all clusters detected in our $\mathrm{X}$-ray survey and for the restricted sample of detected clusters within the Shapley redshift range. The cluster differential luminosity function is defined as:

$\Phi\left(L_{\mathrm{X}}, z\right)=\frac{\mathrm{d}^{2} N}{\mathrm{~d} V \mathrm{~d} L_{\mathrm{X}}}\left(L_{\mathrm{X}}, z\right)$

where $N$ is the number of clusters of luminosity $L_{\mathrm{X}}$ at a redshift $z$ and within a volume $V$. For our sample we compute a binned (with 5 clusters per bin) luminosity function:

$\Phi\left(L_{\mathrm{X}}, z\right)=\frac{1}{\Delta L} \sum_{i=1}^{N} \frac{1}{V_{\max }\left(L_{\mathrm{X}, i}\right)}$

where the sum over $N$ is the sum over all the clusters falling into the luminosity interval of the bin and $V_{\max }$ is the co-moving volume in which a cluster of luminosity $L_{\mathrm{X}, i}$ could have been detected above the flux limit of our survey. Details on how to compute the luminosity functions are given by Böhringer et al. (2002). The top panel of Fig. 11 shows the resulting binned XLF for all clusters detected in our survey in the $0.5-2.0 \mathrm{keV}$ energy range ( $X L F$-All, black points). To allow a direct comparison with previous surveys the XLFs are computed for an Einstein-de Sitter cosmology $\left(\Omega_{\mathrm{M}}=1, \Omega_{\Lambda}=0\right)$ with $H_{0}=$ $50 \mathrm{~km} \mathrm{~s}^{-1} \mathrm{Mpc}^{-1}$. The XLFs estimated from the Brightest Cluster Sample (BCS+eBCS) (Ebeling et al. 1998, 2000), REFLEX (Böhringer et al. 2001) and RASS1BS (De Grandi et al. 1999) surveys are plotted as solid, dotted and dashed lines, respectively. All three XLFs from the literature provide an acceptable fit to our data, for clusters with luminosities higher than $2 \times 10^{43} \mathrm{erg} \mathrm{s}^{-1}$; a slight excess of low luminosity clusters is observed in our survey.

In the bottom panel we construct a tentative LF from our survey for the Shapley SC alone (XLF-Shapley). To this aim we use a restricted sub-sample from our survey, given by exclusively clusters which lie in the Shapley redshift range $(0.03 \leq z \leq 0.07)$. We also add the clusters detected in the second step analysis. These two samples are obtained with different flux limits. We hence compute two XLFs-Shapley using two different flux limits: the luminosity of the faintest cluster detected in our analysis and the flux limit of our survey. The respective XLFs (plotted with empty circles and filled triangles) can therefore be regarded as "upper" and "lower limit" 


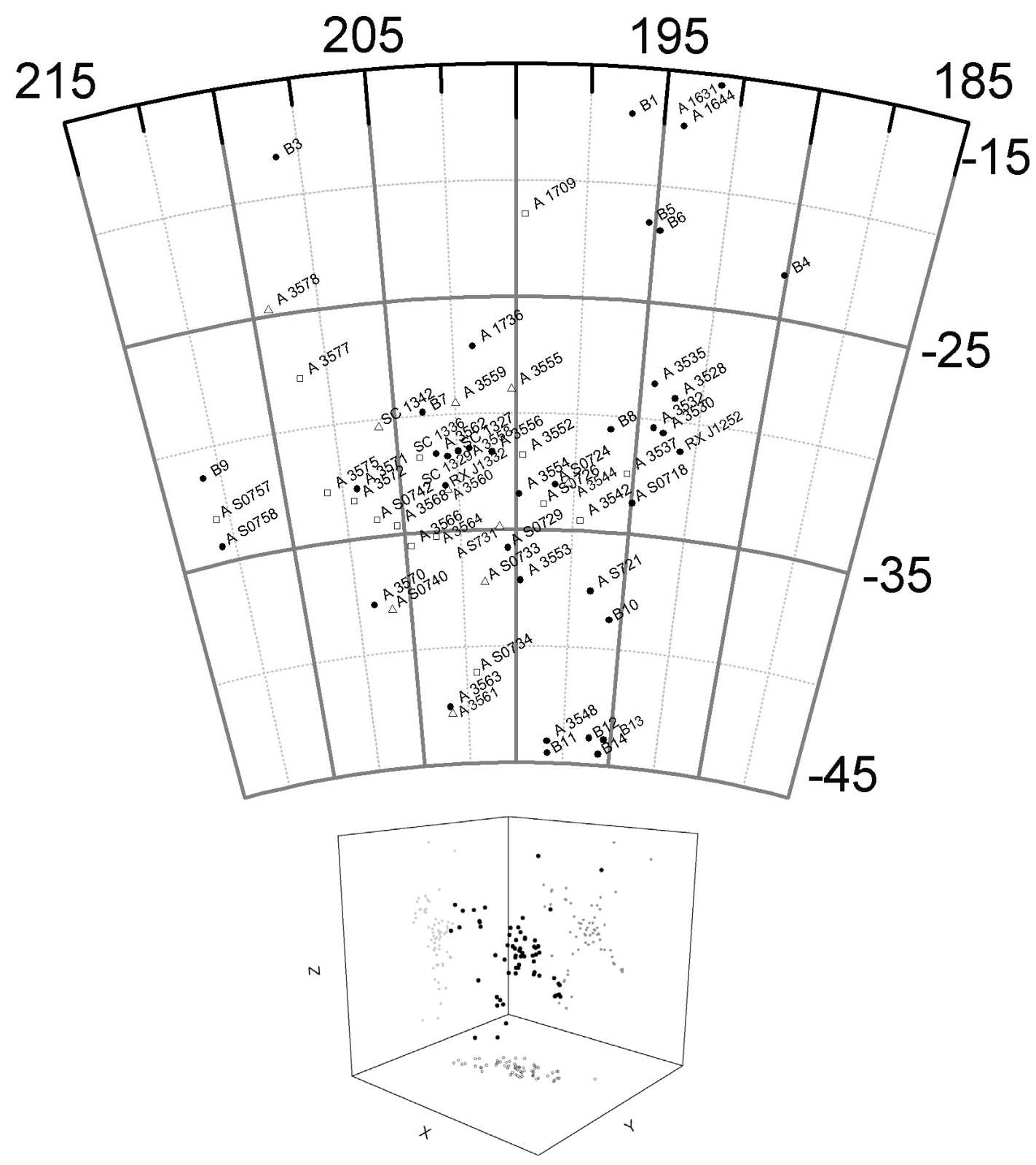

Fig. 10. Positions of all known clusters in the area inside the redshift range $0.03 \leq z \leq 0.07$. Top: RA vs. Dec; filled circles indicate clusters selected and detected in this work, empty squares represent the clusters selected during the second step analysis while empty triangles give the positions of all the optically known clusters, which have not been detected in this survey. Bottom: three-dimensional orthogonal projection; redshift is along the $z$ axis.

for the XLF-Shapley. The two dotted lines represent their bestfits. They are both much steeper than values in literature: $\alpha_{\mathrm{L}}=$ $2.4, \Phi_{\mathrm{L}}^{*}=3.0 \times 10^{-8}\left(h_{50}^{3} \mathrm{Mpc}^{-3}\right), L_{\mathrm{L}}^{*}=5.7 \times 10^{44}\left(h_{50}^{-2} \mathrm{erg} \mathrm{s}^{-1}\right)$ and $\alpha_{\mathrm{U}}=2.15, \Phi_{\mathrm{U}}^{*}=3.7 \times 10^{-8}\left(h_{50}^{3} \mathrm{Mpc}^{-3}\right), L_{\mathrm{U}}^{*}=5.7 \times$ $10^{44}\left(h_{50}^{-2} \mathrm{erg} \mathrm{s}^{-1}\right)$. Even if this restricted sub-sample can definitely not be considered complete, and therefore no quantitative result can be drawn from it, we use it to draw generic conclusions on the luminosity population of the clusters in the Shapley SC. In Sect. 8 we measured an over-abundance of clusters in the Shapley region, and especially in the core, with respect to the average number density of Abell clusters. We can now say that while the bright cluster population of the whole Shapley SC is consistent with what is observed in lower density environments, low luminosity clusters are over-abundant; low luminosity clusters are hence the main cause of the observed excess in the cluster number density of this super-structure.

If we restrict our analysis to the central dense core of the SC alone $(0.0446 \leq z \leq 0.0554,193.14 \leq \mathrm{RA} \leq 208.74$ and $-37.64 \leq$ Dec $\leq-27.15$, plotted as empty squares in the bottom panel of Fig. 11), while we still observe an over-abundance of low-luminosity objects, high luminosity clusters also are in excess with respect to the background distribution. Since we expect strongly interacting and merging clusters to be X-ray luminous objects, because the X-ray luminosity is enhanced during a merger, the external regions of the SC are most probably still in a process of accreting low luminosity, small objects through the surrounding filaments. Major close encounters, at 

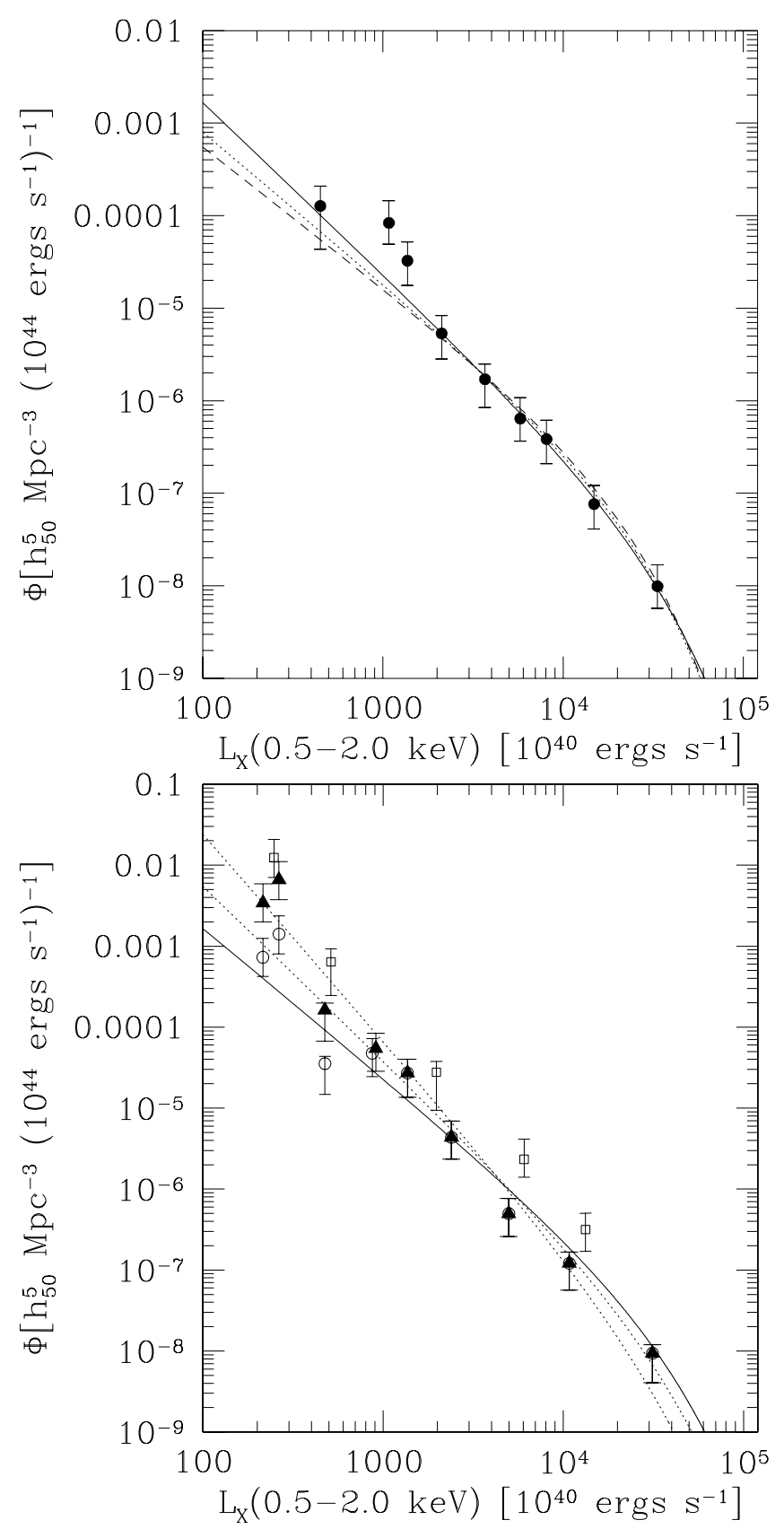

Fig. 11. X-ray luminosity function. Top: black points represent the binned XLF of our survey. The solid, dotted and dashed lines are the XLFs computed for the BCS, REFLEX and RASS1BS surveys. Bottom: "lower" and "upper" limit (see text) for the XLF of all the clusters detected in the Shapley redshift range (empty circles and filled triangles, respectively); the two dotted lines are the two best-fit results. The XLF for the central core of the SC is plotted as empty squares. The solid black line is as defined in the top panel.

this point of the SC formation, affect the luminosity of the cluster population only in the central densest core.

\subsection{Optical versus $X$-ray cluster properties}

From Girardi et al. $(2000,1998)$ we extracted the values of the optical luminosities in the $B_{j}$ band computed within the
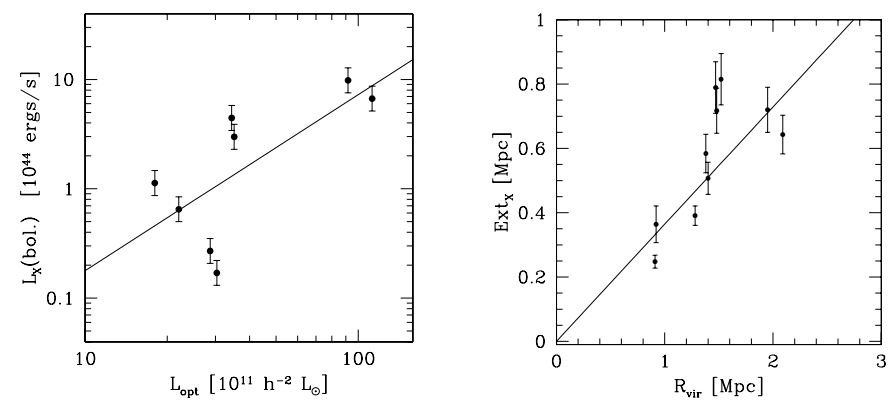

Fig. 12. Left panel: bolometric X-ray luminosity versus optical luminosity. Right panel: extension of the cluster from our X-ray detection versus virial radius. Best-fit estimates are plotted as solid lines.

cluster virial radius, together with the values of the virial radii available for the clusters in our sample.

In the left panel of Fig. 12 we plot the bolometric X-ray luminosity of the clusters versus their optical $B_{j}$ luminosity. The solid line represents the result of the fit:

$L_{\mathrm{X}}=10^{(-2.36 \pm 1.09)} L_{\mathrm{opt}}^{(1.61 \pm 0.68)}$.

In the right panel is the extent of the clusters as computed from our detection algorithm against their virial radius, both in units of Mpc. The best-fit line is plotted as a solid line.

$\operatorname{Ext}_{\mathrm{X}}=(0.36 \pm 0.14) \cdot R_{\mathrm{vir}}$.

For both relations there are signs of a trend, but the number of objects in our sample for which optical data are available is unfortunately too low to be able to give any definite result. Similar, more accurate trends for the $L_{\mathrm{X}}-L_{\mathrm{opt}}$ relation, based on a much larger sample, were recently found by Popesso et al. (2005).

\subsection{Merger rates/cosmology}

The dynamical state of clusters of galaxies depends on the matter density $\Omega_{\mathrm{m}}$. This was first shown in an analytical approach by Richstone et al. (1992) and modified later by other groups taking into account time variations for substructure to be erased (Kauffmann \& White 1993; Lacey \& Cole 1993; Nakamura et al. 1995). As the amount of substructure is expected to increase with $\Omega_{\mathrm{m}}$, several X-ray samples have been used to determine the frequency of substructure (i.e. the merger rate): Mohr et al. (1995) 50-70\%; Jones \& Forman (1999) 40\%; Schuecker et al. (2001) $52 \pm 7 \%$. The results show some scatter in the numbers because different methods have been used and the rates also depend on the resolution of the instrument as well as on several other parameters.

The merger rates, however, do not only depend on the overall mass density but they are expected to depend also on the local density.

To determine the merger rate for the Shapley super-cluster we classified the clusters according to their morphology. 25 clusters have sufficient photons to determine the morphology; to these we added the four new cluster detection which were confirmed clusters in the optical (B1, B4, B5 and B9). In the last column of Table 4 these clusters are flagged with an 
"M" if they show any signature of mergers (i.e. from clearly interacting structures, to strongly irregular, filamentary and unsymmetrical and deformed morphologies). We find that out of the 29 clusters 22 clusters show such signatures, i.e. a fraction of $76 \%$ of the clusters are not relaxed. We are aware that such a classification is just a somewhat arbitrary estimate, but we believe it to be sufficient for our actual purpose, which is to compare it with several previous estimates, which were all derived with different methods.

Obviously, the merger rate in the Shapley super-cluster is higher than in all the general cluster samples by Mohr et al. (1995); Jones \& Forman (1999); Schuecker et al. (2001). This confirms the expectations of an increasing merger rate with increasing local density, which also has been found by Schuecker et al. (2001). This dependence provides a possibility to determine the total mass of the Shapley super-cluster in an independent way. From simulations the merger rate could be determined at different local mass densities. With the same method the merger rate could be determined from an observed general cluster sample and from our Shapley sample. The comparison would yield the total mass density and hence also the total mass of the Shapley super-cluster. We hope that such a set of simulations will be available soon.

\section{The cumulative mass profile}

We then compute the cumulative mass profile of all detected clusters within the restricted Shapley redshift range $(0.0446 \leq$ $z \leq 0.0554$ ); this is defined as the measured (from X-rays) matter distribution in the Shapley SC as a function of the comoving 3-D distance from A 3558. The total mass for each cluster is estimated from their X-ray luminosity using the $L_{\mathrm{X}} / M$ relation by Reiprich \& Böhringer (2002):

$\log \left[\frac{L_{\mathrm{X}}(0.1-2.4 \mathrm{keV})}{h_{50}^{-2} \mathrm{erg} \mathrm{s}^{-1}}\right]=A+\alpha \log \left(\frac{M_{200}}{h_{50}^{-1} M_{\odot}}\right)$

with $\alpha=1.807 \pm 0.084$ and $A=-22.053 \pm 1.251$. The cumulative mass profile is then estimated by summing the masses of all clusters detected within the selected Shapley redshift range (including clusters detected in the second step analysis). In Fig. 13 we compare the resulting mass profile, plotted as black dots, with the expected virial and cosmic masses, in a similar way as done by Ettori et al. (1997). The dashed line represents the expected mass, within the same distance from A 3558, in a homogeneous Universe of density $\rho_{\mathrm{c}} \Omega_{\mathrm{m}}$ :

$M_{0}=\frac{H_{0}^{2}}{2 G} R^{3} \Omega_{0}(1+z)^{3}$.

The empty triangles represent the values of the virial mass computed considering the single clusters as test particles using:

$M_{\mathrm{Vir}}=\frac{3 \pi}{G} \frac{\sigma^{2}}{\left\langle 1 / R_{\perp i, j}\right\rangle}$

where $\sigma$ is the standard deviation of the distribution of the observed velocities of the clusters and $\left\langle 1 / R_{\perp i, j}\right\rangle$ is the harmonic mean radius. Table 3 lists the values of the total X-ray mass $\left(M_{\mathrm{X}}\right)$, of the mass expected within a homogeneous Universe

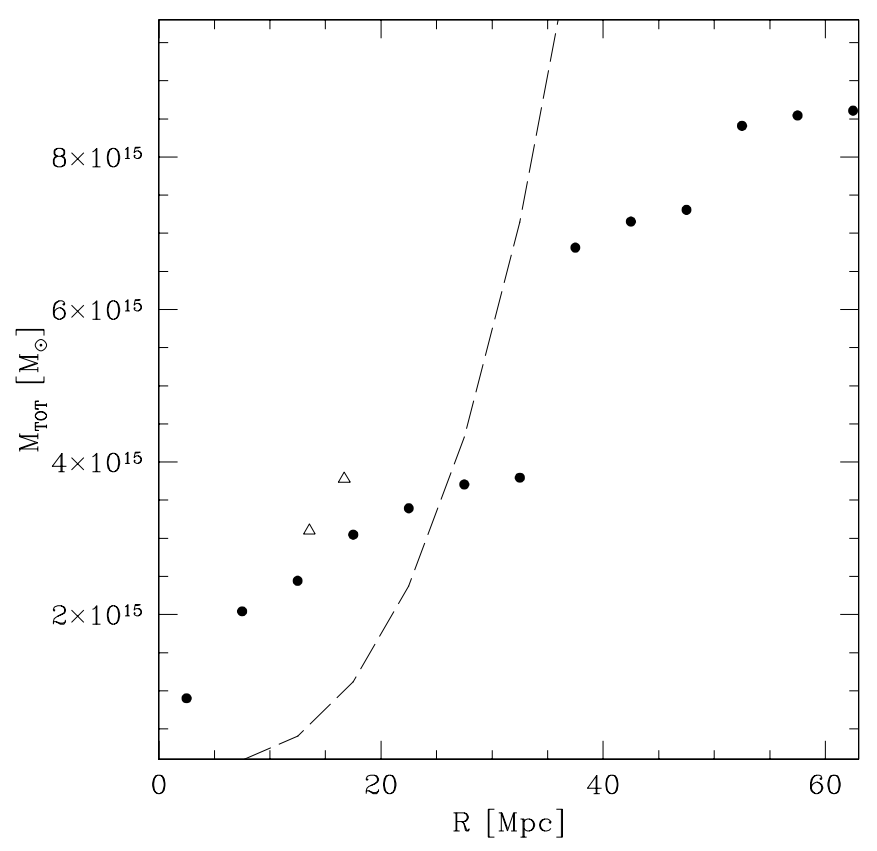

Fig. 13. Cumulative mass profile of detected clusters in the Shapley redshift range (black dots). The dashed line represents the expected total mass in a homogeneous Universe of density $\rho_{\mathrm{c}} \Omega_{0}$. The empty triangles are the values of the virial mass computed considering the single clusters as test particles. See Table 3 for numerical values at different radii.

Table 3. Total mass estimates.

\begin{tabular}{ccccc}
\hline \hline $\begin{array}{c}R \\
(\mathrm{Mpc})\end{array}$ & $N_{\mathrm{cl}}$ & $\begin{array}{c}M_{\mathrm{X}} \\
\left(10^{14} M_{\odot}\right)\end{array}$ & $\begin{array}{c}M_{0} \\
\left(10^{14} M_{\odot}\right)\end{array}$ & $\begin{array}{c}M_{\text {Vir }} \\
\left(10^{14} M_{\odot}\right)\end{array}$ \\
\hline 13.6 & 7 & 23.5 & 5.2 & 31.0 \\
16.7 & 9 & 28.8 & 9.7 & 37.8 \\
40.0 & 28 & 57.7 & 133.2 & 173.6 \\
60.8 & 31 & 72.3 & 467.9 & 180.9 \\
\hline
\end{tabular}

Col. (1): Co-moving distance from A 3558 within which $M_{\mathrm{X}}, M_{0}$ and $M_{\text {vir }}$ (Cols. (3)-(5), respectively) are estimated. $M_{\mathrm{X}}$ is computed as the sum of the masses of all clusters detected in the Shapley redshift range (including clusters detected in the second step analysis). In Col. (2) is the number of clusters analyzed within each radius.

$\left(M_{0}\right)$ and of the virial mass $\left(M_{\mathrm{Vir}}\right)$, computed within four increasing radii. While we do measure an over-abundance of X-ray mass up to $R=25 \mathrm{Mpc}$ with respect to $M_{0}$, the gravitating mass is still slightly lower than the virial value. This does not come as a surprise since the SC has not yet reached a virial equilibrium (Ettori et al. 1997; Bardelli et al. 2000) and seems to be still accreting matter through the surrounding filamentary large scale structure (see Sect. 8).

Our X-ray cumulative mass profile is strictly a lower limit since it is computed considering only the sum of the gas and gravitating mass of each cluster. We neglect any contribution from a possible intra-supercluster-medium and dark matter and do not consider matter in the outer regions of clusters, where the X-ray emission detected in the RASS survey gets confused with the background. Furthermore, some of the known optical clusters are still undetected in X-rays; this could be due to the 
fact that these clusters are chance alignments of galaxies along the line of sight, or that they represent extremely faint structures, below our flux limit.

As seen in Sect. 9, the Shapley SC shows an extremely rich population of faint extended sources; this population provides a substantial portion of the mass content of the whole SC. We hence stress the necessity to explore this region, as well as other similarly crowded ones, to increasingly lower X-ray luminosities in order to obtain an exhaustive knowledge of their mass and of their cluster population.

We estimate the total mass of the SC using all clusters detected in the Shapley redshift range. To this aim we use the $X L F$-Shapley measured in Sect. 9 to compute the total $L_{\mathrm{X}}$ emitted by the SC. We then estimate the total SC X-ray mass using Eq. (7). If we include clusters as faint as those detected in our second step analysis $\left(L_{\mathrm{X}} \approx 2 \times 10^{42} \mathrm{erg} \mathrm{s}^{-1}\right)$ the resulting SC total mass range is $M_{\mathrm{X}}=5-8 \times 10^{15} M_{\odot}$ (from the lower and upper limit of the $X L F$-Shapley). Integrating to even lower luminosities, including structures as faint as $L_{\mathrm{X}} \approx 1 \times 10^{42} \mathrm{erg} \mathrm{s}^{-1}$, would further double the value of the total SC mass.

\section{Results and discussion}

We have surveyed the X-ray emission of a large area surrounding the Shapley SC using RASS-III data. To this purpose we used a detection algorithm, explicitly written to detect nearby extended, interacting and/or irregular sources. Within the analyzed region our algorithm initially detected $56 \%$ of all optically known clusters below redshifts of 0.07 . For 9 of them this was the first X-ray detection. Only 3 clusters with previous X-ray detection were not found in our survey. For each optically known cluster which was not detected, we performed a more accurate second step analysis lowering our flux limit. In this way we could detect X-ray emission from $40 \mathrm{SC}$ members already known in the optical ( $\sim 80 \%$ of all known extended structures); for half of these clusters this is the first detection in $\mathrm{X}$-rays. We also isolated 14 new cluster candidates. Follow-up observations have shown at least 4 of these candidates have optical counterparts at their X-ray positions.

The analysis of such a wide area has allowed us to study the cluster distribution not only in the SC core but also in its surroundings. The overall structure of the SC looks slightly flattened and elongated; showing an extremely dense core at the center $(193.14 \leq \mathrm{RA} \leq 208.74,-37.64 \leq \mathrm{Dec} \leq-27.15$ and $0.0446 \leq z \leq 0.0554)$. The clusters surrounding the dense core lie along perpendicular filaments similarly to what is shown by large scale structure simulations; they are most probably moving towards the SC core, attracted by its gravitational potential.

Compared to previous X-ray analysis of the region (Raychaudhury et al. 1991; Ettori et al. 1997), we have largely increased the number of clusters in the area detected in X-rays (thanks to both the wide area analyzed and the low flux limit reached in our second step analysis). This has led to an estimate of the local cluster number density more than one order of magnitude higher than previous optical estimates (Scaramella et al. 1989) both in the core region and in the much less dens surroundings.
In our measure of the X-ray mass we kept a strictly conservative approach, since we neglected any contribution from baryonic and dark matter between the clusters and from the outer regions of the clusters. In the SC core we measure an over-abundance of X-ray mass with respect to what expected in a homogeneous Universe, while the mass in clusters is slightly lower than what is needed to account for the virial value, sign that the SC, even in its central core, is not yet virialized.

A direct comparison with previous works is not straightforward because of several fundamental differences which differentiate each work (i.e. number of clusters, sky region, cosmology, etc.). As a general statement we can though say that our $\mathrm{X}$-ray mass estimate is consistent with that measured by Ettori et al. (1997) within a distance of $20 \mathrm{Mpc}$ from A 3558, and higher only up to a factor of 1.6 going out to larger radii. Our estimates is though based on a higher number of clusters; at the same time Ettori et al. (1997) extrapolate the cluster mass out to much larger radii. Both X-ray values barely reach the lower limits of the gravitating mass estimated by Raychaudhury et al. (1991); Quintana et al. (1995) and Bardelli et al. (2000) using different approaches (excess in the number of galaxies, virial estimate of each cluster mass, etc.). Once deep X-ray observations of a larger number of Shapley clusters will be available, we will be able to obtain more accurate mass measures, out to increasingly larger radii, even for the faintest clusters in the region.

Rich, X-ray luminous clusters are common objects in the central dense SC core (Raychaudhury et al. 1991). The analysis of a wide sky area performed in this work has highlighted that these are instead much more rare objects in the SC outskirts. Faint clusters and poor groups are instead over-abundant both in the central and in the outer regions of the Shapley cluster region compared to what is measured in less dense environments. Such objects are hence expected to provide an extremely important addition to the SC mass content. To date we have examined only the luminous tail of the X-ray luminosity function for the Shapley region. We therefore underline the importance of exploring these crowded regions to fainter luminosities, which would significantly increase our knowledge of their mass and population content.

Acknowledgements. This paper is based on observations obtained from the ROSAT Public Data Archive and on observations made with ESO Telescopes at the La Silla Observatory. We thank MPE and ESO for maintaining their archives active and running and the NASA HPCC ESS group at the University of Washington for making publicly available their software tools. This work has been supported by NASA grants NAS8-39073, by FWF grant P15868 of the Austrian Science Foundation and by the Deutsche Forschungsgemeinschaft (DFG) under the project ER 327/2-1.

We thank the referee Stefano Ettori for his help and useful comments.

\section{References}

Abell, G. O., Corwin, H. G., \& Olowin, R. P. 1989, ApJS, 70, 1 Böhringer, H., Collins, C. A., Guzzo, L., et al. 2002, ApJ, 566, 93 Böhringer, H., Schuecker, P., Guzzo, L., et al. 2004, A\&A, 425, 367 Böhringer, H., Schuecker, P., Guzzo, L., et al. 2001, A\&A, 369, 826 Bardelli, S., Zucca, E., Vettolani, G., et al. 1994, MNRAS, 267, 665 
Bardelli, S., Zucca, E., Malizia, A., et al. 1996, A\&A, 305, 435

Bardelli, S., Zucca, E., Zamorani, G., Vettolani, G., \& Scaramella, R. 1998, MNRAS, 296, 599

Bardelli, S., Zucca, E., Zamorani, G., Moscardini, L., \& Scaramella, R. 2000, MNRAS, 312, 540

Bertin, E., \& Arnouts, S. 1996, A\&AS, 117, 393

Bonamente, M., Lieu, R., Nevalainen, J., \& Kaastra, J. S. 2001, ApJ, 552, L7

Bruzual A., G., \& Charlot, S. 1993, ApJ, 405, 538

Burstein, D., Davies, R. L., Dressler, A., Faber, S. M., \& Lynden-Bell, D. 1986, in Galaxy Distances and Deviations from Universal Expansion, NATO ASIC Proc., 180, 130

Calzetti, D., Armus, L., Bohlin, R. C., et al. 2000, ApJ, 533, 682

David, L. P., Slyz, A., Jones, C., et al. 1993, ApJ, 412, 479

David, L. P., Forman, W., \& Jones, C. 1999, ApJ, 519, 533

Day, C. S. R., Fabian, A. C., Edge, A. C., \& Raychaudhury, S. 1991, MNRAS, 252, 394

De Filippis, E. 2003, Ph.D. Thesis, Astrophysics Research Institute, Liverpool John Moores University, UK

De Grandi, S., Böhringer, H., Guzzo, L., et al. 1999, ApJ, 514, 148

Dickey, J. M., \& Lockman, F. J. 1990, ARA\&A, 28, 215

Ebeling, H., Voges, W., Böhringer, H., et al. 1996, MNRAS, 281, 799

Ebeling, H., Edge, A. C., Böhringer, H., et al. 1998, MNRAS, 301, 881

Ebeling, H., Edge, A. C., Allen, S. W., et al. 2000, MNRAS, 318, 333

Einasto, M., Einasto, J., Tago, E., Dalton, G. B., \& Andernach, H. 1994, MNRAS, 269, 301

Einasto, M., Tago, E., Jaaniste, J., Einasto, J., \& Andernach, H. 1997, A\&AS, 123, 119

Erben, T., Schirmer, M., Dietrich, J. P., et al. 2005, AN, 326, 432

Ettori, S., Fabian, A. C., \& White, D. A. 1997, MNRAS, 289, 787

Ettori, S., Bardelli, S., De Grandi, S., et al. 2000, MNRAS, 318, 239

Gastaldello, F., Ettori, S., Molendi, S., et al. 2003, A\&A, 411, 21

Giavalisco, M., Ferguson, H. C., Koekemoer, A. M., et al. 2004, ApJ, 600, L93

Girardi, M., Giuricin, G., Mardirossian, F., Mezzetti, M., \& Boschin, W. 1998, ApJ, 505, 74

Girardi, M., Borgani, S., Giuricin, G., Mardirossian, F., \& Mezzetti, M. 2000, ApJ, 530, 62

Gonzalez, A. H., Zaritsky, D., Dalcanton, J. J., \& Nelson, A. 2001, ApJS, 137, 117

Hanami, H., Tsuru, T., Shimasaku, K., et al. 1999, ApJ, 521, 90
Hasinger, G., Burg, R., Giacconi, R., et al. 1993, A\&A, 275, 1

Jarrett, T. H., Chester, T., Cutri, R., et al. 2000, AJ, 119, 2498

Jenkins, A., Frenk, C. S., Pearce, F. R., et al. 1998, ApJ, 499, 20

Jones, C., \& Forman, W. 1999, ApJ, 511, 65

Kauffmann, G., \& White, S. D. M. 1993, MNRAS, 261, 921

Lacey, C., \& Cole, S. 1993, MNRAS, 262, 627

Markevitch, M. 1998, ApJ, 504, 27

Markevitch, M., \& Vikhlinin, A. 1997, ApJ, 474, 84

Markevitch, M., Forman, W. R., Sarazin, C. L., \& Vikhlinin, A. 1998, ApJ, 503, 77

Mohr, J. J., Evrard, A. E., Fabricant, D. G., \& Geller, M. J. 1995, ApJ, 447, 8

Mullis, C. R., Vikhlinin, A., Henry, J. P., et al. 2004, ApJ, 607, 175

Nakamura, F. E., Hattori, M., \& Mineshige, S. 1995, A\&A, 302, 649

Nevalainen, J., Kaastra, J., Parmar, A. N., et al. 2001, A\&A, 369, 459

Niklas, S., Klein, U., Braine, J., \& Wielebinski, R. 1995, A\&AS, 114, 21

Popesso, P., Biviano, A., Böhringer, H., Romaniello, M., \& Voges, W. 2005, A\&A, 433, 431

Quintana, H., Melnick, J., Proust, D., \& Infante, L. 1997, A\&AS, 125, 247

Quintana, H., Ramirez, A., Melnick, J., Raychaudhury, S., \& Slezak, E. 1995, AJ, 110, 463

Raychaudhury, S., Fabian, A. C., Edge, A. C., Jones, C., \& Forman, W. 1991, MNRAS, 248, 101

Reid, A. D., Hunstead, R. W., \& Pierre, M. M. 1998, MNRAS, 296, 531

Reiprich, T. H., \& Böhringer, H. 2002, ApJ, 567, 716

Richstone, D., Loeb, A., \& Turner, E. L. 1992, ApJ, 393, 477

Scaramella, R., Baiesi-Pillastrini, G., Chincarini, G., Vettolani, G., \& Zamorani, G. 1989, Nature, 338, 562

Schindler, S. 1996, MNRAS, 280, 309

Schirmer, M., Erben, T., Schneider, P., et al. 2003, A\&A, 407, 869

Schuecker, P., Böhringer, H., Reiprich, T. H., \& Feretti, L. 2001, A\&A, 378, 408

Venturi, T., Bardelli, S., Morganti, R., \& Hunstead, R. W. 1997, MNRAS, 285, 898

Voges, W., Aschenbach, B., Boller, T., et al. 1999, A\&A, 349, 389

Wu, X., Xue, Y., \& Fang, L. 1999, ApJ, 524, 22

Yoshida, N., Colberg, J., White, S. D. M., et al. 2001, MNRAS, 325, 803 


\section{Online Material}


E. De Filippis et al.: The Shapley super-cluster, Online Material p 2

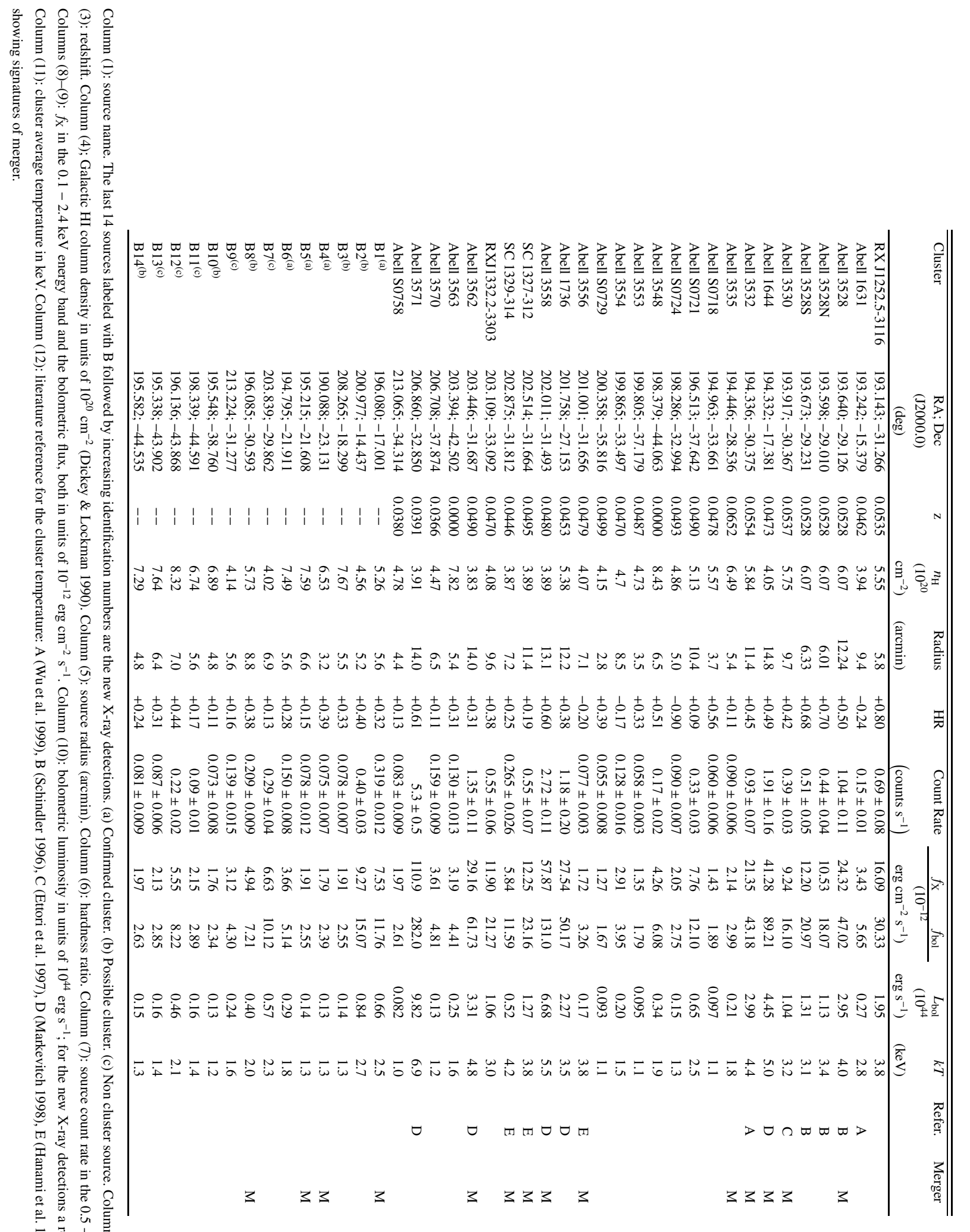




\section{Appendix A: Optical properties of B1, B4-B9}

\section{and B11-B14}

In this Appendix we discuss the analysis of the optical data for our new Shapley SC candidates B1, B4-B9 and B11-B14 (see Sect. 7 and Table 4). We extract object catalogs from our $B$ and $R$ WFI images by using SExtractor (Bertin \& Arnouts $1996)$ in the double image mode, i.e. we use the deeper $R$-band image to detect objects (we considered all sources having at least 4 pixels with $2 \sigma$ over the sky background noise) and determined aperture magnitudes in $R$ and $B$ (we used a fixed aperture with a diameter of 6.' 0 ) around these positions. Figure A.1 explains how we separate stars and galaxies and in which area of our color-magnitude diagrams we search for a Red Cluster Sequence of potential Shapley SC members. With our selection criteria, the galaxy number counts start to drop at $R \approx 23$ which should be sufficient for the detection of massive galaxy clusters at $z \approx 0.05$. The results of our analysis of all fields is shown in Figs. A.2-A.15. 
E. De Filippis et al.: The Shapley super-cluster, Online Material p 4
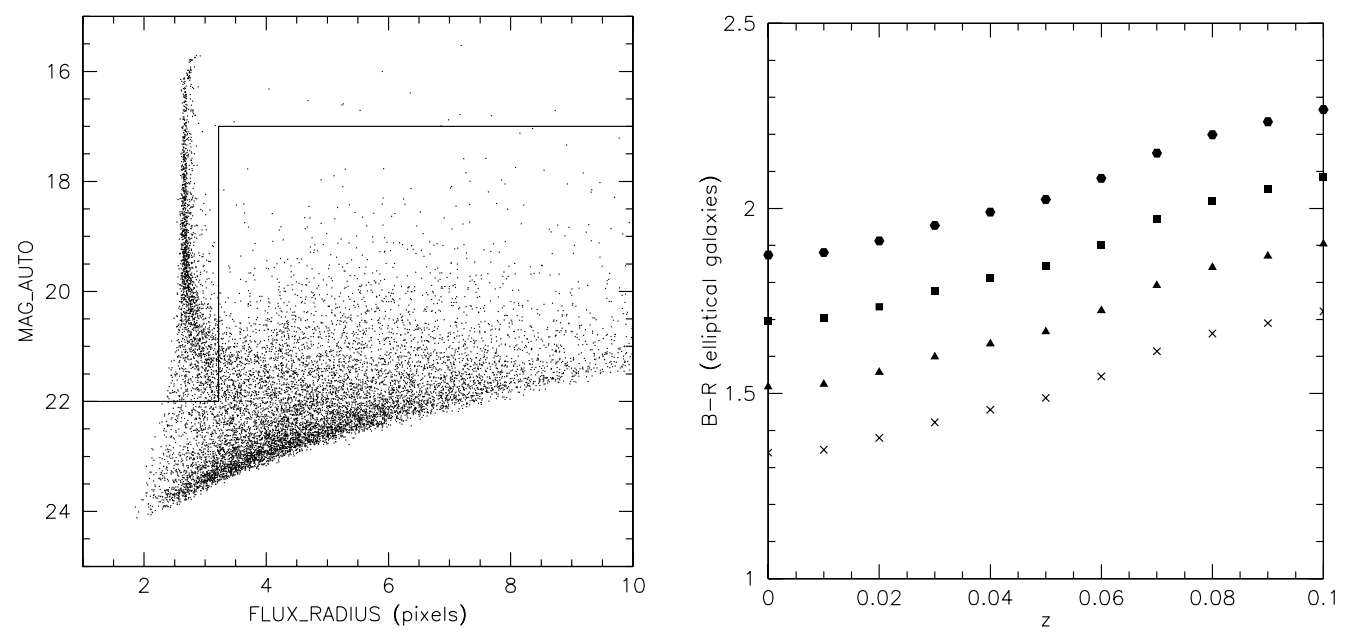

Fig. A.1. Shown in the left panel is a half-light radius (SExtractor FLUX_RADIUS parameter) vs. $R$ magnitude (SExtractor MAG_AUTO parameter) diagram from all detected objects in the field of B5. The bright stars are located in a narrow locus around $r_{\mathrm{h}} \approx 3.2$. In our analysis we reject all objects in the box shown around the stellar locus and we apply an additional cut for bright galaxies $(R<17)$. The contamination of our galaxy catalog with stars in the faint part of the stellar locus should not affect our analysis significantly. We reject bright objects to avoid the domination of our light maps by individual galaxies. Identical plots were created and examined for all fields (the cut for bright objects is always at $R=17$ ). The right panel shows $B-R$ colors of elliptical galaxies as a function of redshift. To create the plot we used galaxy templates from Bruzual \& Charlot (1993) with an exponential law (time-scale $1 \mathrm{Gyr}$ ) for the star formation rate. We applied the reddening law from Calzetti et al. (2000) with extinctions $A_{\mathrm{v}}=0.0$ (crosses), $A_{\mathrm{v}}=0.5$ (triangles), $A_{\mathrm{v}}=1.0$ (squares) and $A_{\mathrm{v}}=1.5$ (hexagons). Given this plot together with our $B$ and $R$ band data we search for Red Cluster Sequences of potential SC members in the color range $1.5<B-R<2$.

\section{B1 (Ro: 13:04:06.16; Dec: $-16: 58: 42)$}

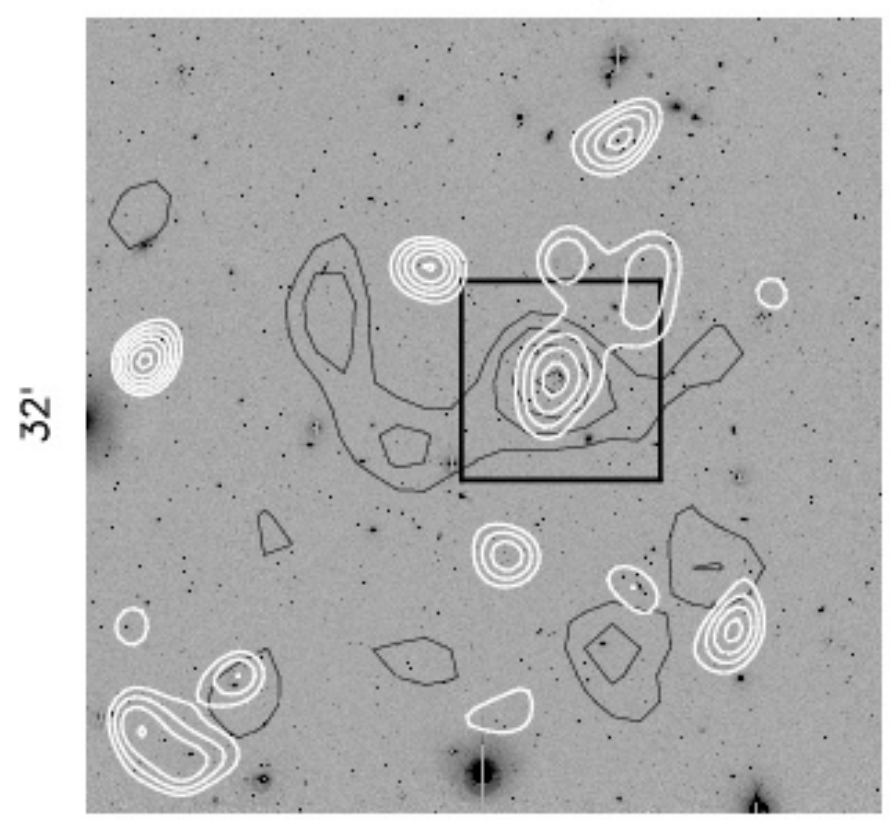

$32^{\prime}$

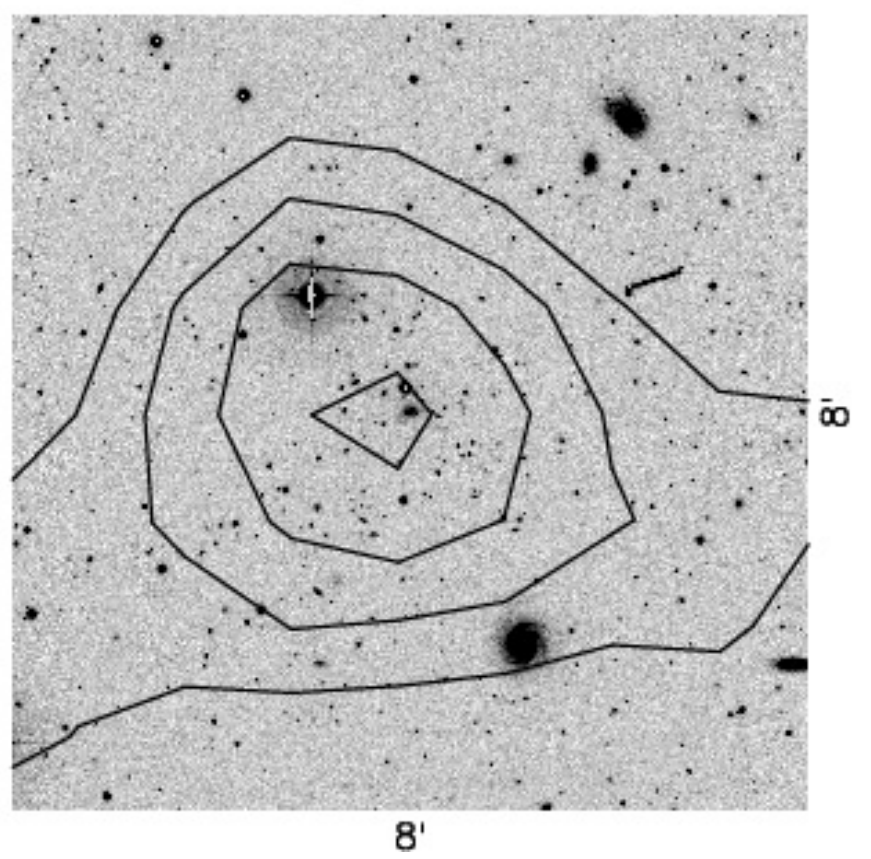

Fig. A.2. Left panel: shown is the full WFI $R$-band field around the X-ray detection B1. RA increases to the left and Dec to the top. Black

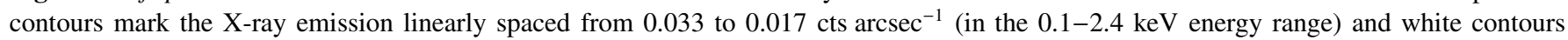
represent 1.5 to $4 \sigma$ (spaced by $0.5 \sigma$ ) optical light over-densities from galaxies above the background level. The light distribution is calculated on a regular grid (spacing 3.2), where each gridpoint contains the total flux within 8'.0 weighted by a Gaussian with a width of 1'.5. Each galaxy with $R>17$ (see also Fig. A.1) is included and the background level and the $\sigma$ are determined from the gridpoints. The black square marks a $8.0 \times 8$ '.0 region around the X-ray peak and is shown enlarged in the right panel ( 8.0 corresponds to $0.46 \mathrm{Mpc}$ at $z=0.05$ ). The X-ray emission is reproduced as black contours. The coordinates in the top label (given in the J2000 system) mark the location of the X-ray peak position. B1 shows a bright X-ray emission and clear light over-density is seen at the X-ray position. In the $B-R$ color space we see an over-density of galaxies at around $B-R \approx 2.2$ (see Fig. A.13) which is very red if it originates from a galaxy cluster at $z \approx 0.05$. We consider this case as a good candidate for a new, previously unidentified galaxy cluster. 
E. De Filippis et al.: The Shapley super-cluster, Online Material p 5

B4 (Ro: 12:40:24.21; Dec: -23:07:38)

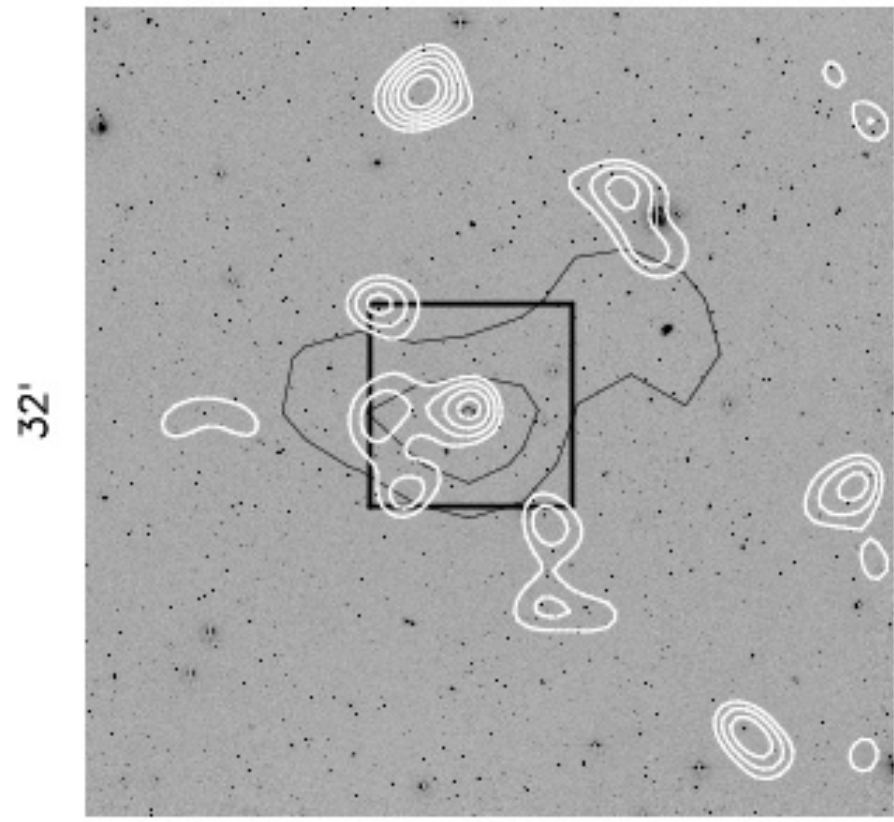

$32^{\prime}$

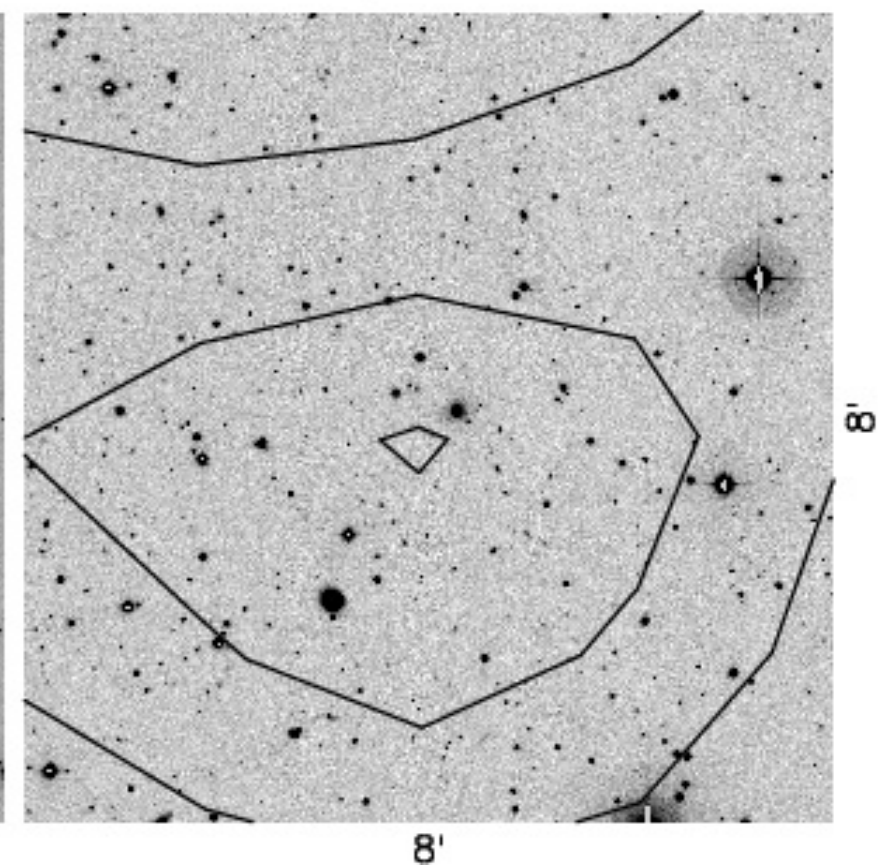

Fig. A.3. Shown are the X-ray and light distributions around our candidate B4. See Fig. A.2 for an explanation of the elements in the figure. A clear light over-density is seen at the X-ray peak position. In the $B-R$ color space we see indications of an over-density of galaxies at around $B-R \approx 1.6-1.8$ (see Fig. A.13). Hence, we consider this case as a good candidate for a new, previously unidentified galaxy cluster.

B5 (Ro: 13:01:02.5; Dec: -21:37:16)

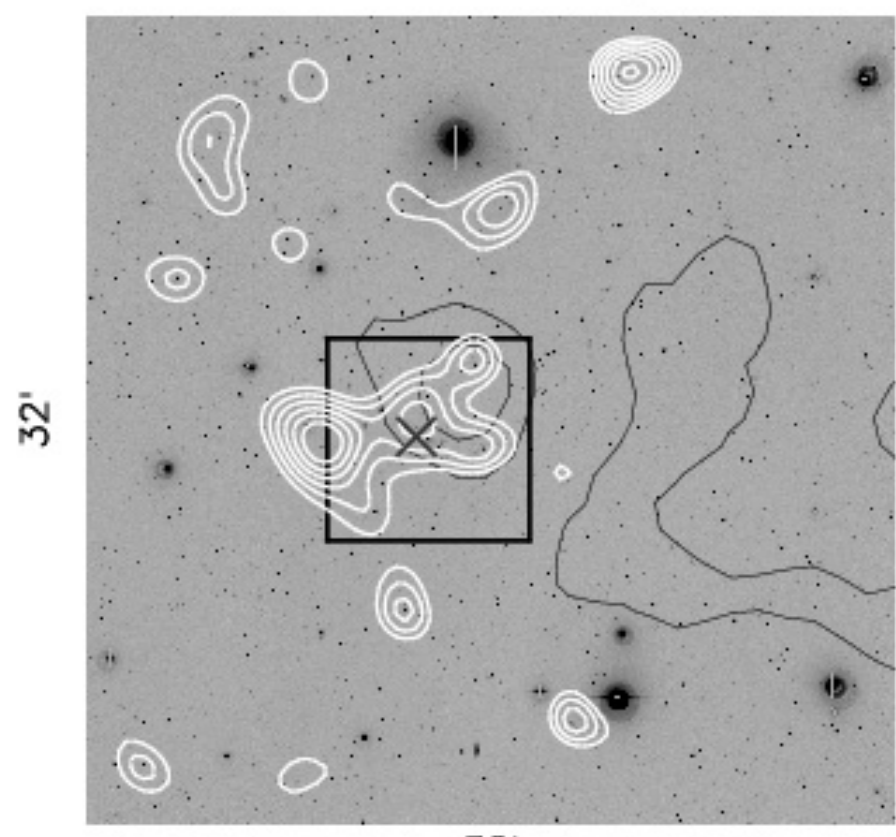

$32^{\prime}$

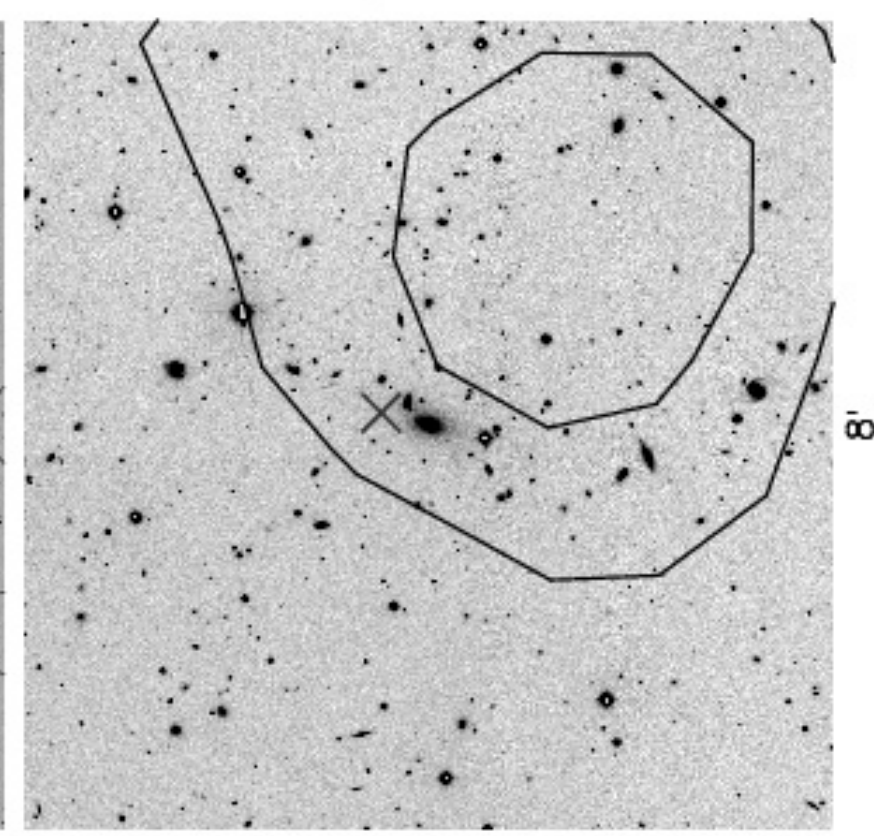

$8^{\prime}$

Fig. A.4. Shown are the X-ray and light distributions around our candidate B5. See Fig. A.2 for an explanation of the elements in the figure. A very strong and elongated light over-density is seen at the $\mathrm{X}$-ray peak position. In the $B-R$ color space we see a clear sequence at $B-R \approx 1.5-1.6$ (see Fig. A.13). In the course of our work we realized that B5 coincides with the position of the galaxy cluster Abell 3538 (marked with a cross in the plots). In in this figure, the $8^{\prime} .0 \times 88^{\prime} .0$ cutout is centered around the coordinates of the Abell cluster. There is no measured redshift for it in the literature. 
B6 (Ro: 12:59:01.6; Dec: -21:54:52)
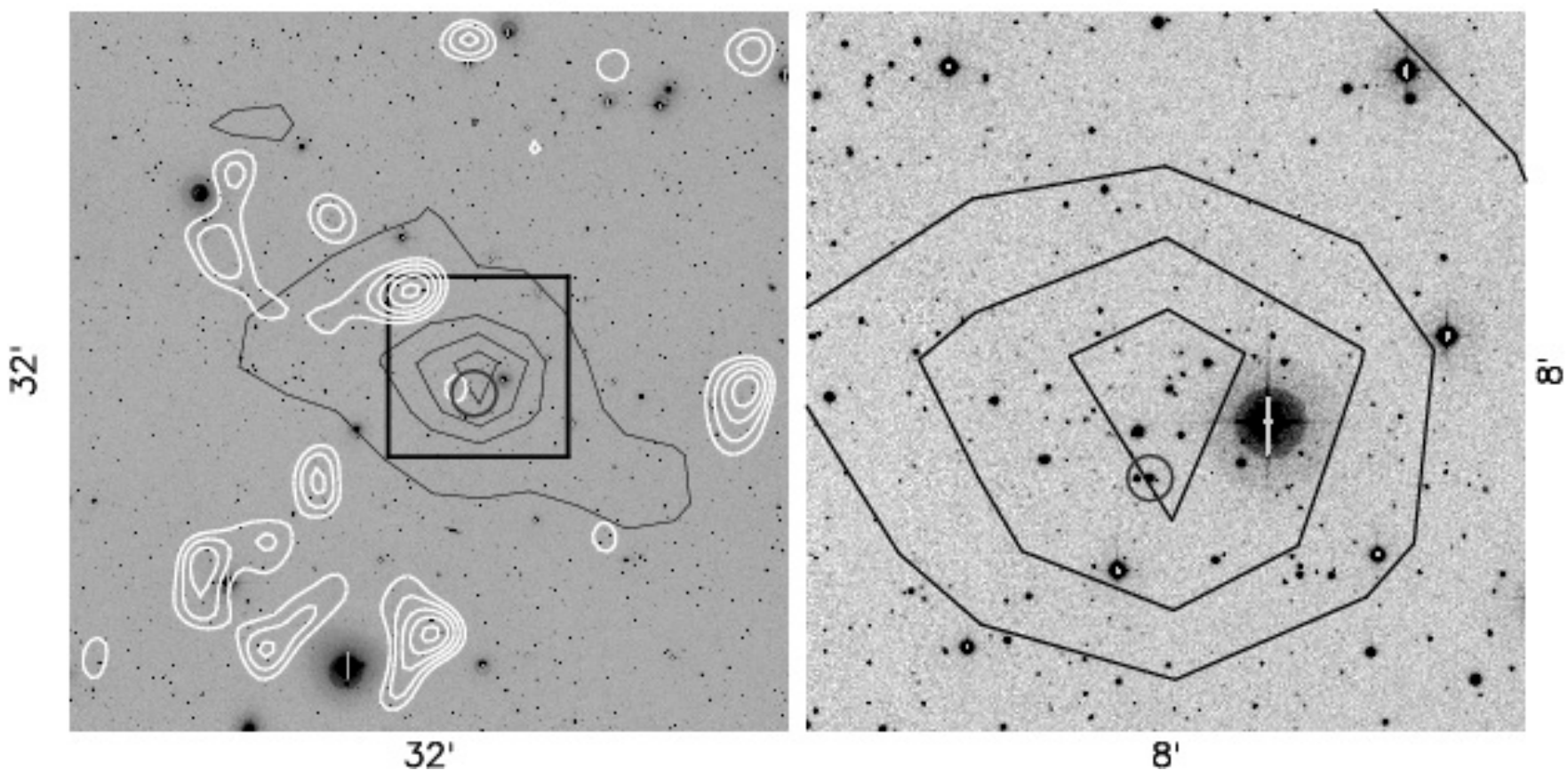

Fig. A.5. Shown are the X-ray and light distributions around our candidate B6. See Fig. A.2 for an explanation of the elements in the figure. B6 has a bright X-ray emission and the primary peak seems to originate from the QSO HE1256-2139 $(z=0.146)$ at 12:59:02.4; $-21: 55: 38$ (J2000) whose position is marked with a circle. However, the X-ray emission is elongated in the east-west direction and we identify a strong light over-density around 12:59:29.86; $-21: 52: 07.4$ (J2000) within the X-ray emission area. In the $B-R$ plot we also see indications for a sequence at around $B-R \approx 1.5-1.7$ (see Fig. A.13). We checked that the sequence does not become more prominent if we include more galaxies to the north-east of the light over-density. We consider B6 as a good candidate for a new, hitherto unidentified galaxy cluster.

\section{B7 (Ro: 13:35:28.4; Dec: $-29: 49: 50)$}

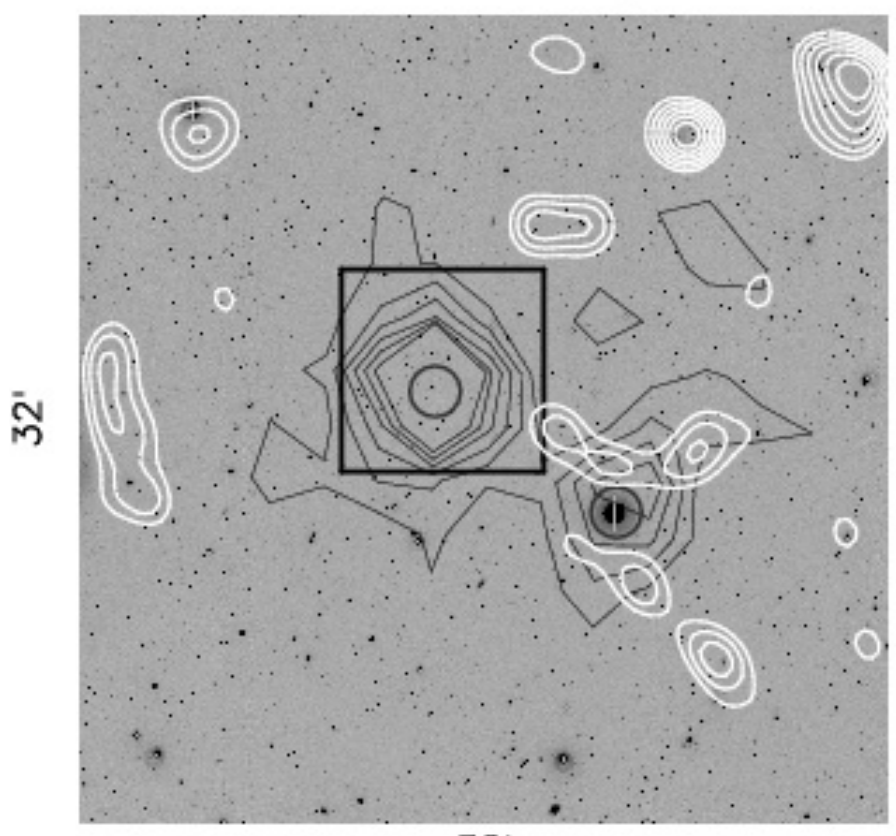

$32^{\prime}$

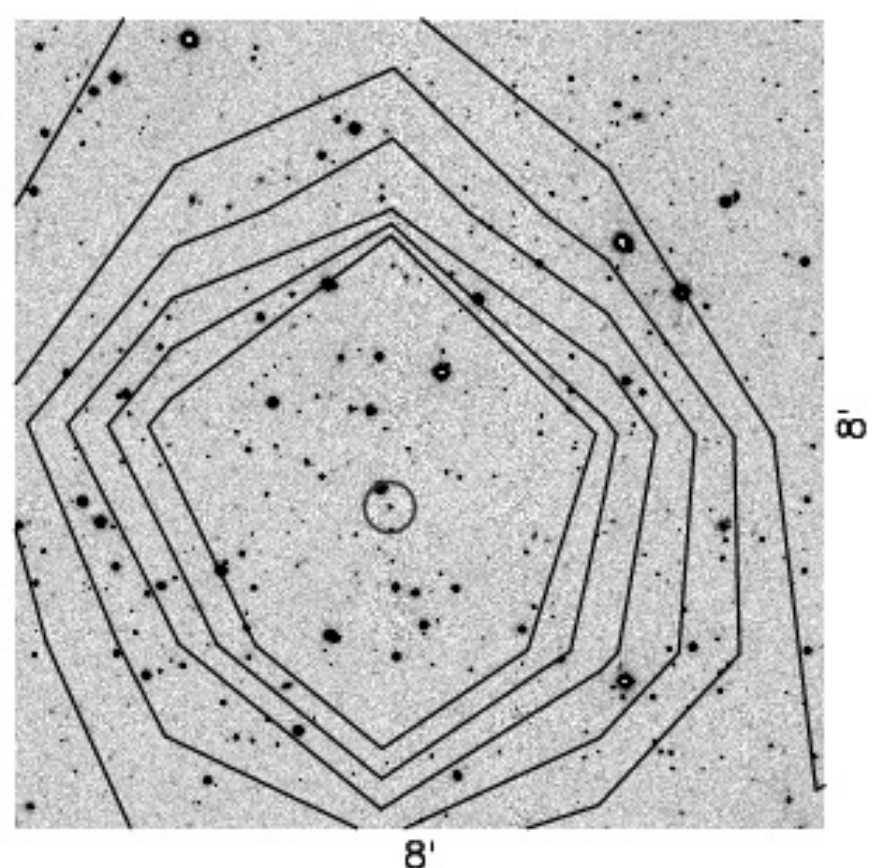

Fig. A.6. Shown are the X-ray and light distributions around our candidate B7. See Fig. A.2 for an explanation of the elements in the figure. B7 shows a double peak X-ray emission that is probably the superposition of the distributions from the QSO RBS1291 $(z=0.25)$ at 13:35:29.7s; $-29: 50: 39 s$ (J2000) and the bright variable star V347 Hya at 13:34:57.40; -29:55:24.0 (J2000). Both point sources are marked with a circle. We see only very slight light over-densities within the X-ray emission and no signs of a sequence in the $B-R$ diagram (see Fig. A.14). Hence, we consider the presence of a new Shapley member in this field as very unlikely. 
B8 (Ro: 13:04:03.6; Dec: -30:28:49)

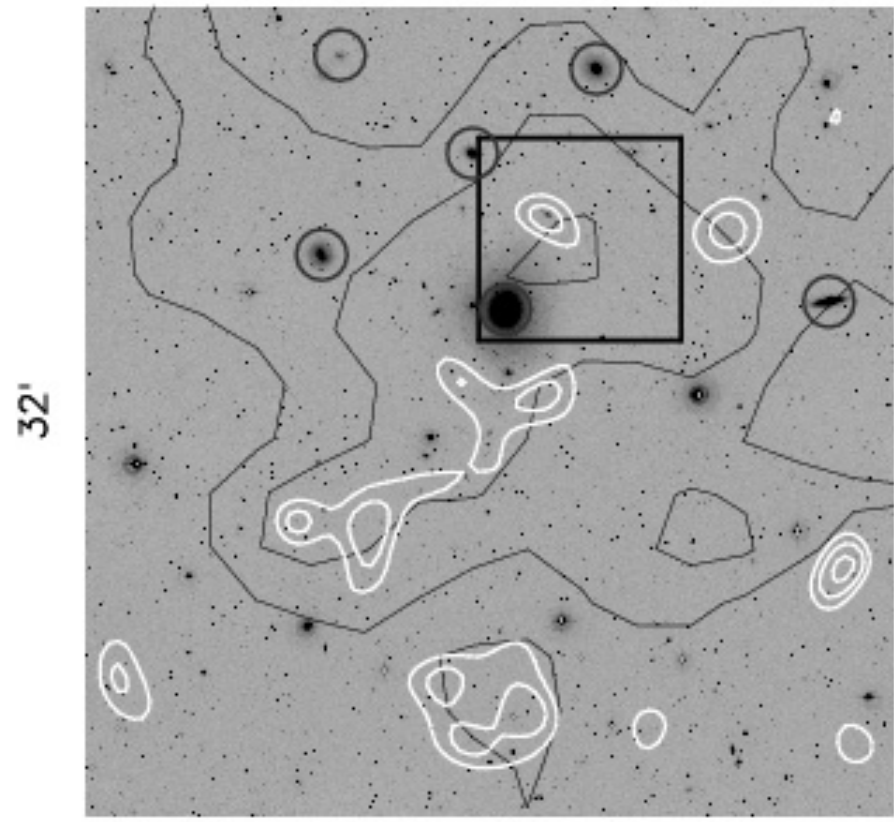

$32^{\prime}$

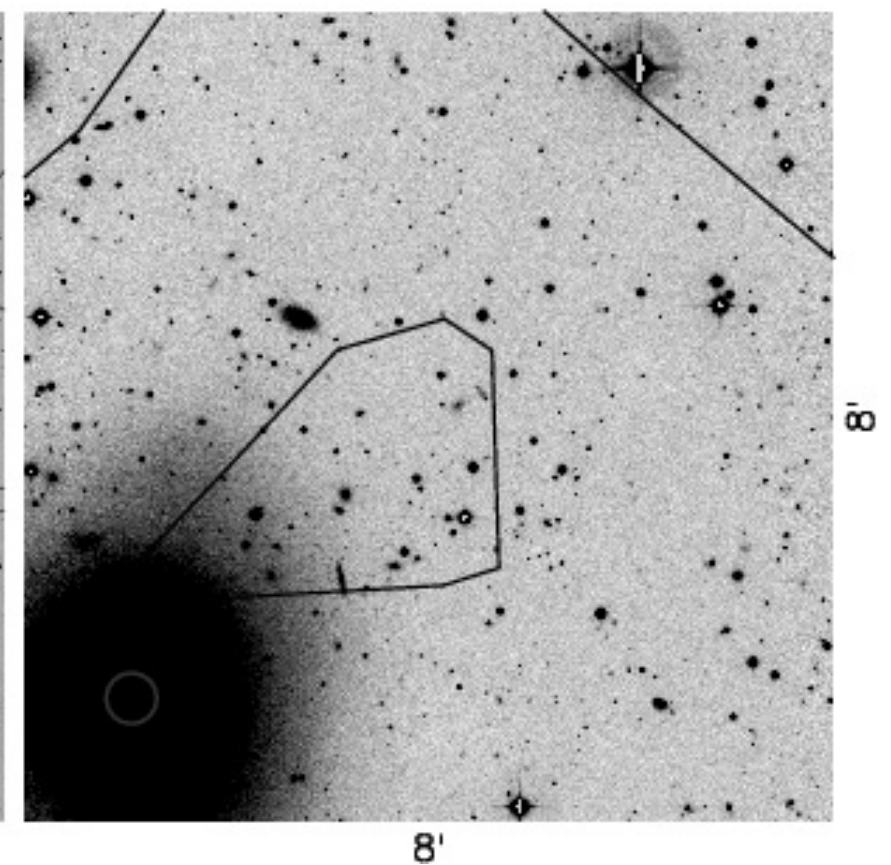

$8^{\prime}$

Fig. A.7. Shown are the X-ray and light distributions around our candidate B8. See Fig. A.2 for an explanation of the elements in the figure. B8 shows a bright, extremely diffuse X-ray emission. We identified several bright galaxies with measured redshifts between 0.0097 and 0.0116 which are marked with circles. Hence, the X-ray flux most probably originates from a galaxy group at $z \approx 0.01$. We see a slight light over-density very close to the $\mathrm{X}$-ray peak but no trends in the $B-R$ diagram although many bright galaxies populate the region between $1.5<B-R<2$ (see Fig. A.14). Hence, we consider this case as uncertain. We note that the X-ray peak position is listed as cluster candidate (RXCJ1304.2-3030) within the REFLEX sample; see Böhringer et al. (2004).

B9 (Ro: 14:13:00.79; Dec: $-31: 15: 52)$

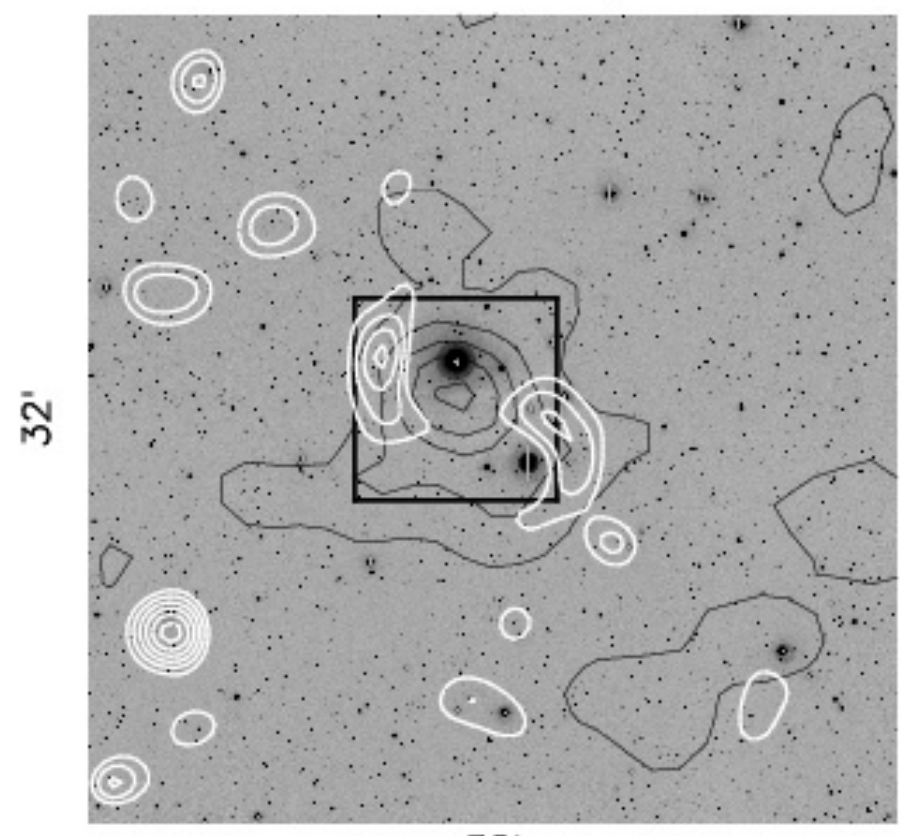

$32^{\prime}$

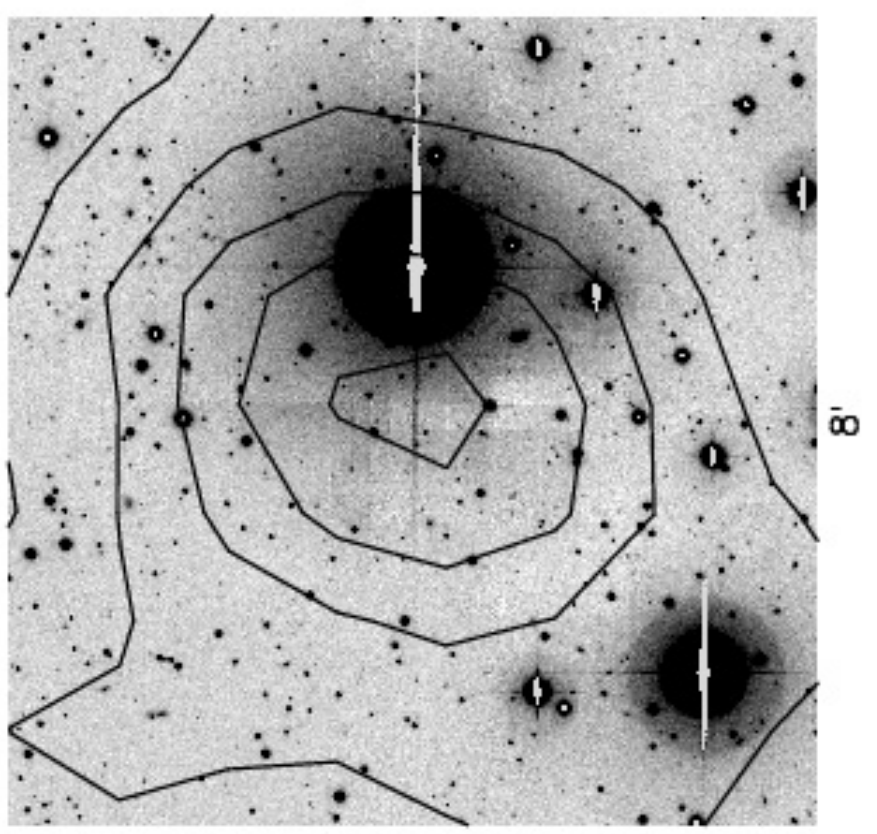

$8^{\prime}$

Fig. A.8. Shown are the X-ray and light distributions around our candidate B9. See Fig. A.2 for an explanation of the elements in the figure. B9 shows a bright X-ray emission. Probably the only source for the observed X-ray emission is the bright star close to the X-ray peak. We see slight galaxy over-densities within the emission area and no signs for galaxy concentrations in the $B-R$ plot (see Fig. A.14). Hence, we consider it unlikely that a Shapley member contributes to the X-ray flux in this field. 
E. De Filippis et al.: The Shapley super-cluster, Online Material $p 8$

B11 (Ro: 13:13:19.46; Dec: $-44: 37: 28$ )

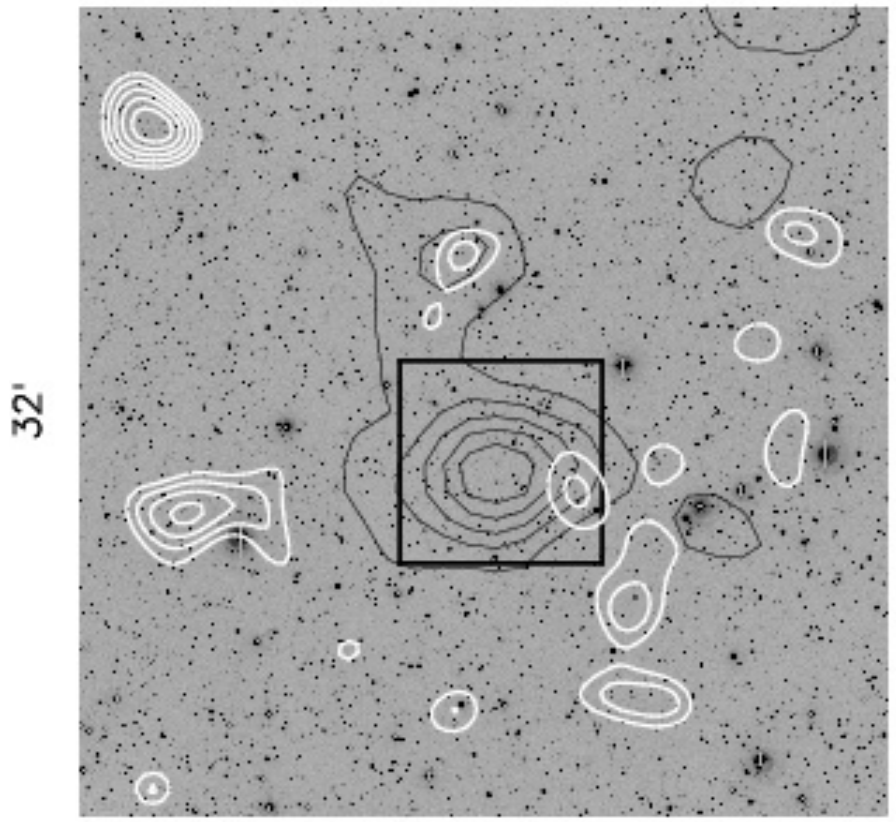

$32^{\prime}$

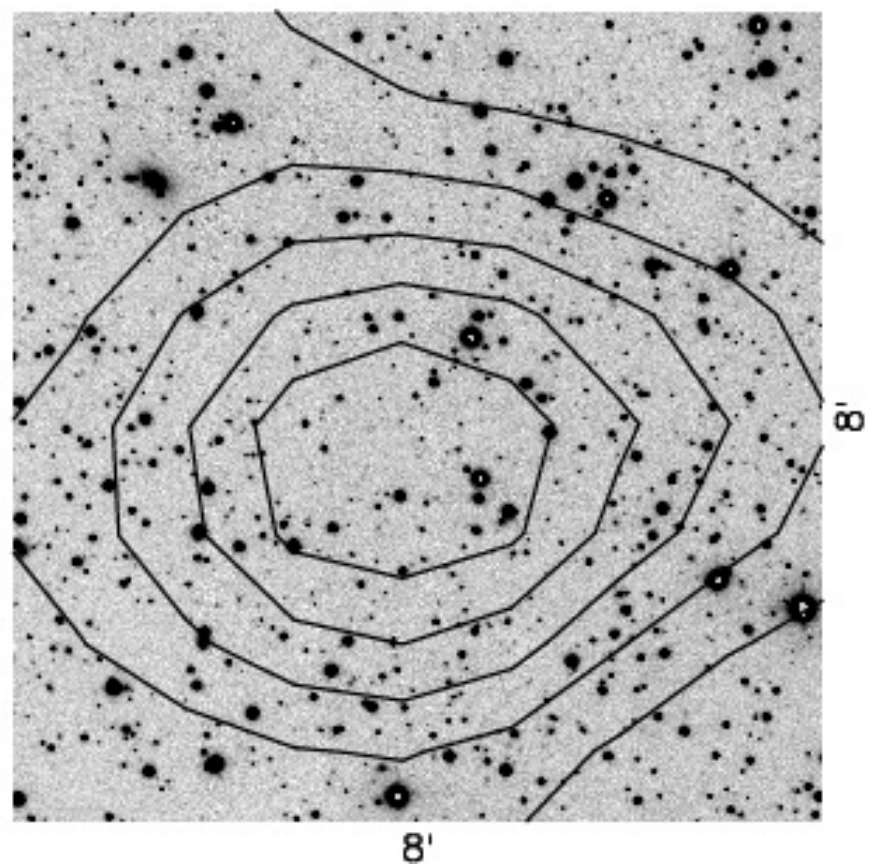

$8^{\prime}$

Fig. A.9. Shown are the X-ray and light distributions around our candidate B11. See Fig. A.2 for an explanation of the elements in the figure. B11 is a field with a high stellar density which significantly hampers the optical search for galaxy over-densities. We see no indications for the possible presence of a galaxy cluster in the light or color $(B-R)$ distributions (see also Fig. A.14). The most probable explanation for the X-ray emission is the superposition of point sources.

B12 (Ro: 13:04:20.59; Dec: $-43: 52: 51)$

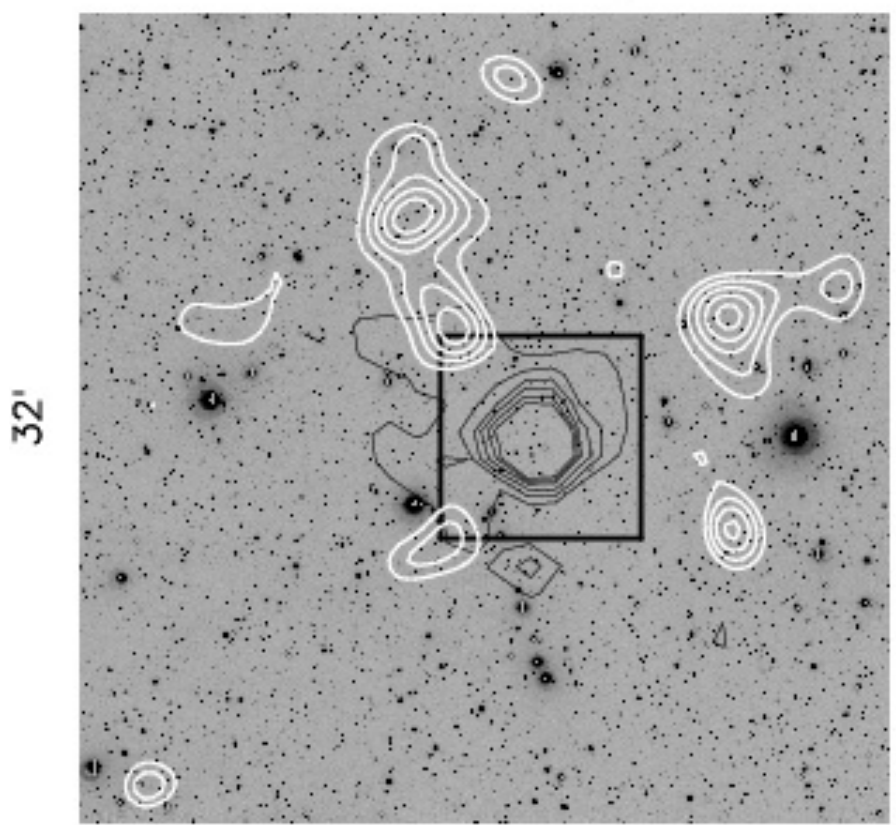

$32^{\prime}$

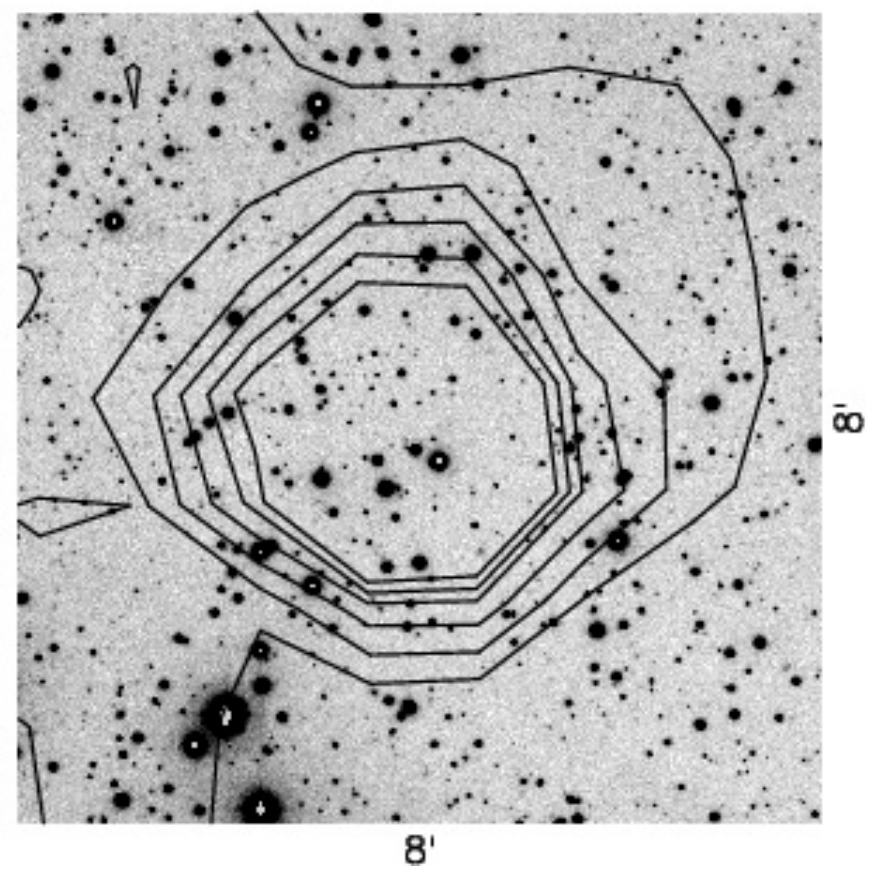

$8^{\prime}$

Fig. A.10. Shown are the X-ray and light distributions around our candidate B12. See Fig. A.2 for an explanation of the elements in the figure. B12 shows a bright an extended X-ray emission. The case of B12 also lies within a stellar field as B11 and our conclusions are the same as for that field. 
E. De Filippis et al.: The Shapley super-cluster, Online Material p 9

B13 (Ra: 13:01:16.24; Dec: -43:57:44)

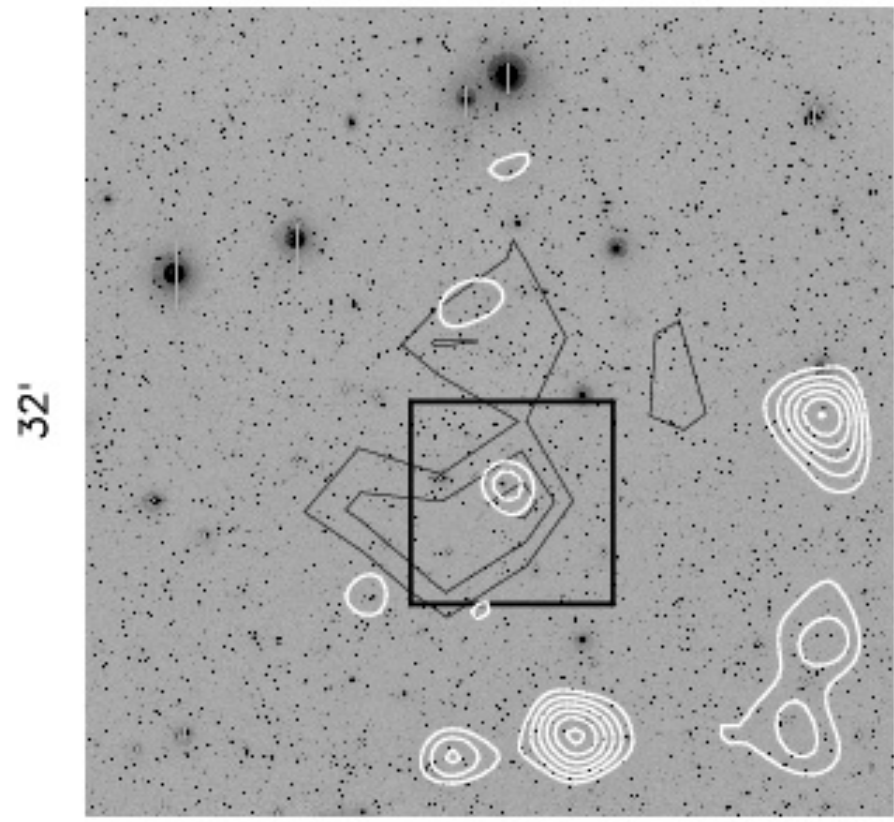

$32^{\prime}$

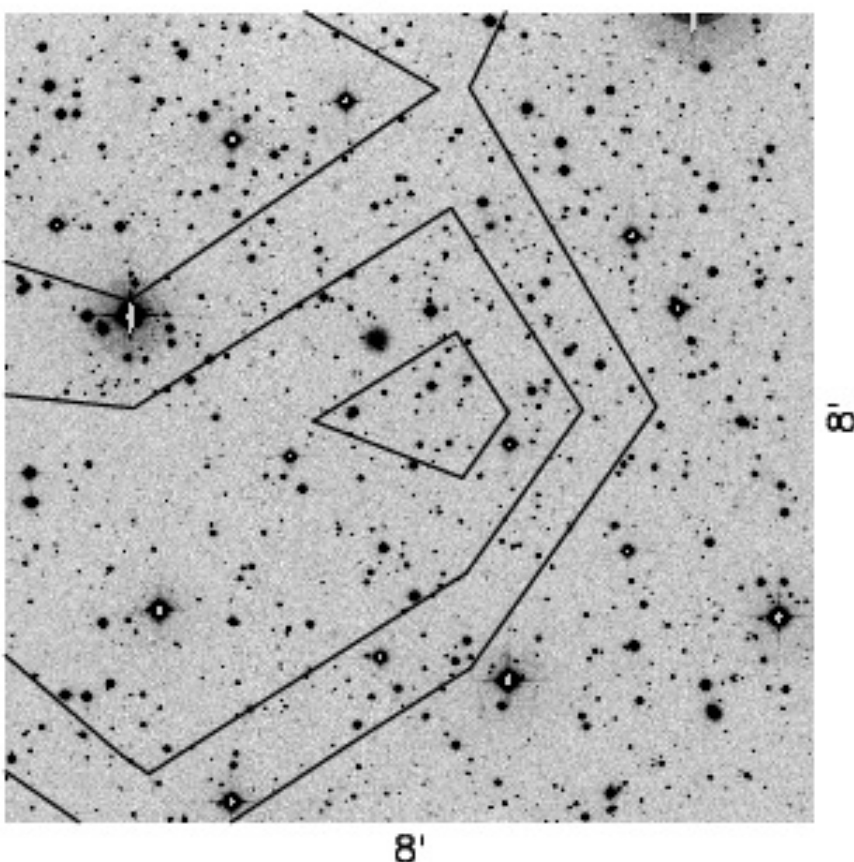

$8^{\prime}$

Fig. A.11. Shown are the X-ray and light distributions around our candidate B13. See Fig. A.2 for an explanation of the elements in the figure. B8 shows an extended X-ray emission. The case of B13 lies within a stellar field as B11 and our conclusions are the same as for that field.

B14 (Ra: 13:02:23.67; Dec: $-44: 32: 49$ )

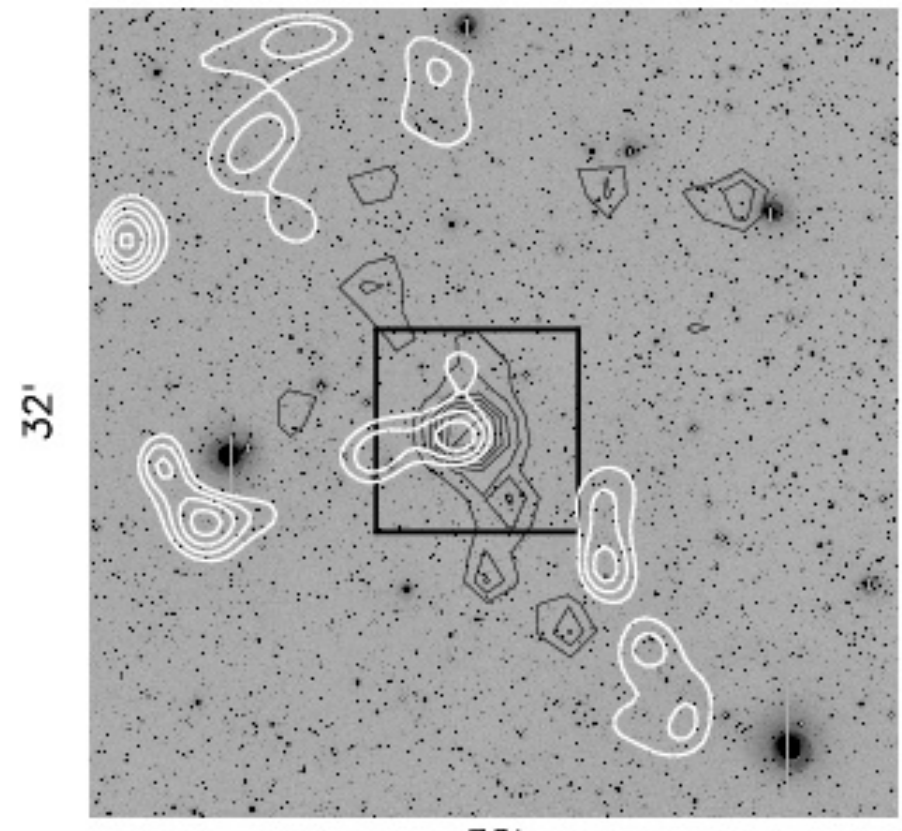

32

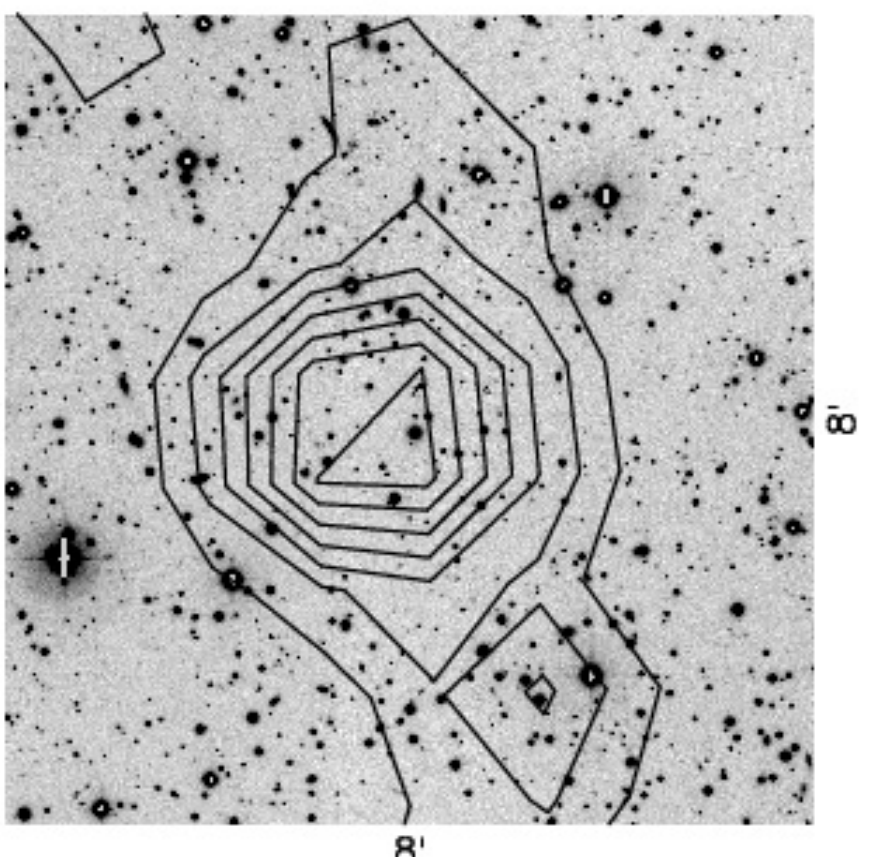

$8^{\prime}$

Fig. A.12. Shown are the X-ray and light distributions around our candidate B14. See Fig. A.2 for an explanation of the elements in the figure. B14 shows an elongated X-ray emission. Similar to B11, B12 and B13 the location within a stellar field makes a quantitative analysis in the optical difficult. Because of the good X-ray detection and an extended over-density directly at the X-ray peak we classify this case as uncertain. No indications for a cluster sequence are seen in the $B-R$ diagram; see Fig. A.15. 
E. De Filippis et al.: The Shapley super-cluster, Online Material p 10

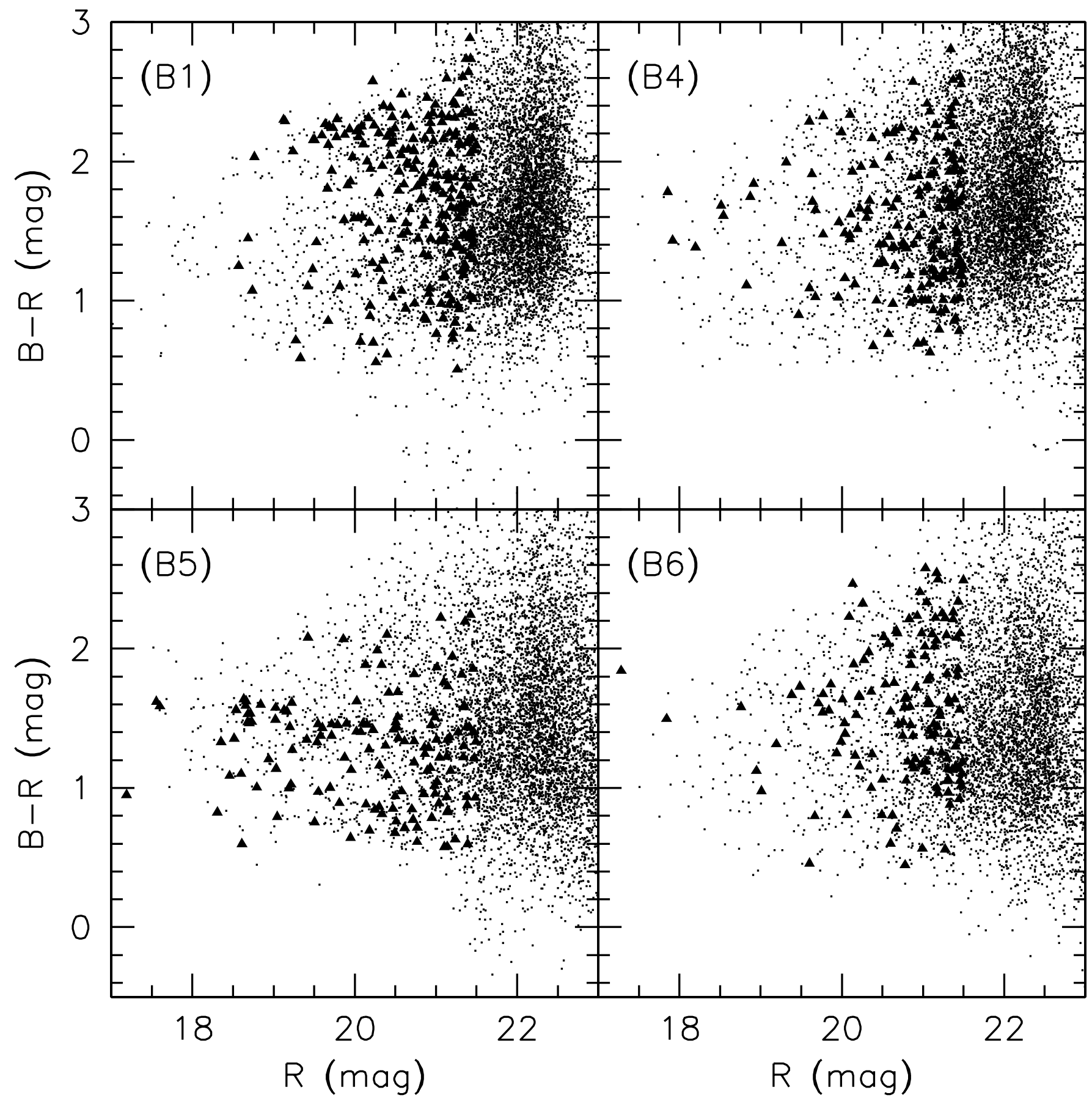

Fig. A.13. Shown are color-magnitude diagrams for our candidates B1, B4, B5 and B6. Dots represent all galaxies within the corresponding WFI fields. Filled triangles represent the galaxies in a $8^{\prime} .0 \times 8^{\prime} .0$ around the X-ray peak positions (see Figs. A.2-A.5). For rich galaxy clusters at $z \approx 0.05$ we would expect a Red-Cluster Sequence from elliptical galaxies around $1.5<B-R<2$ (see also Fig. A.1). 
E. De Filippis et al.: The Shapley super-cluster, Online Material p 11

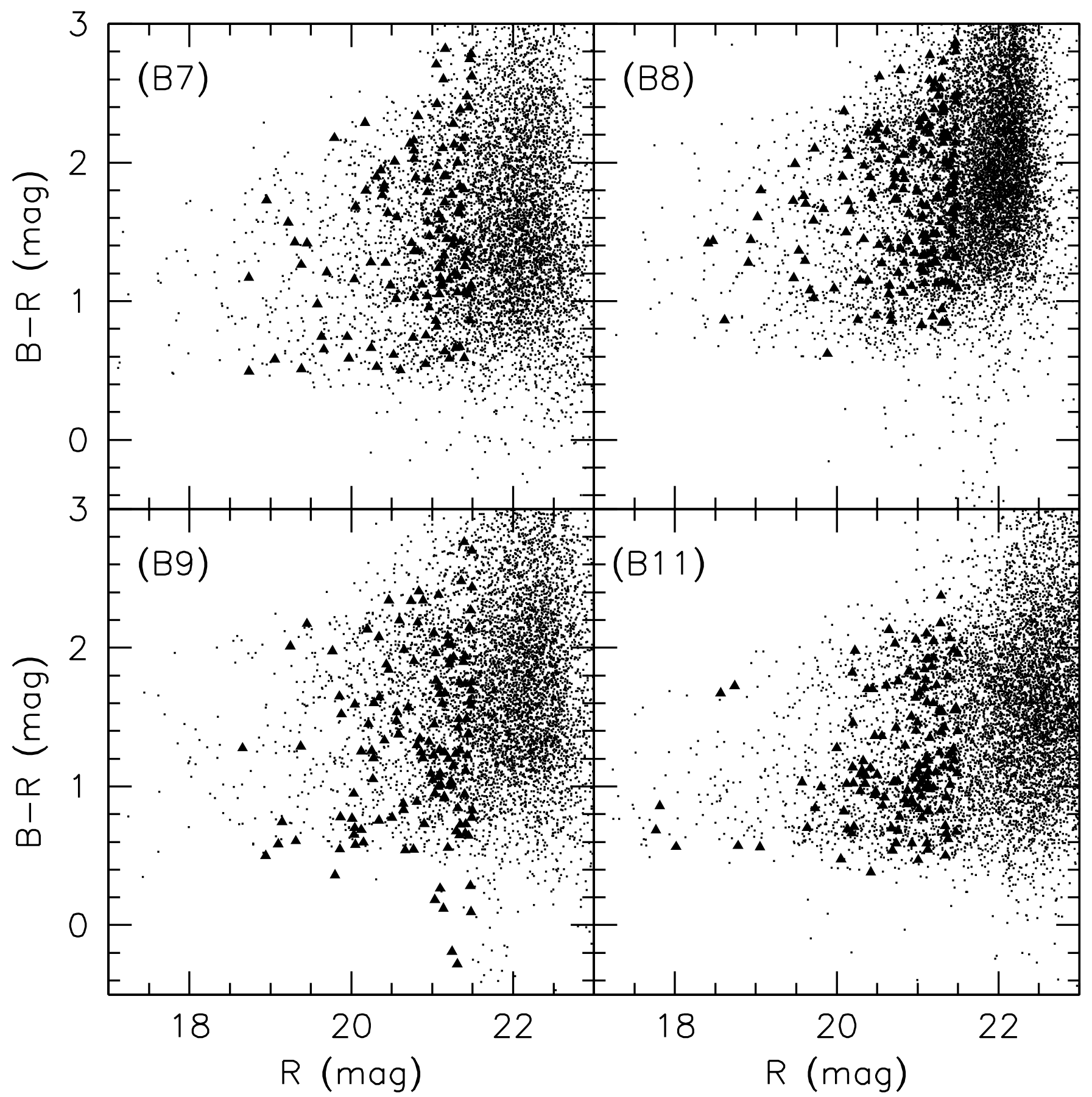

Fig. A.14. Shown are color-magnitude diagrams for our candidates B7, B8, B9 and B11. For an explanation of the plot see the caption from Fig. A.13. 
E. De Filippis et al.: The Shapley super-cluster, Online Material p 12

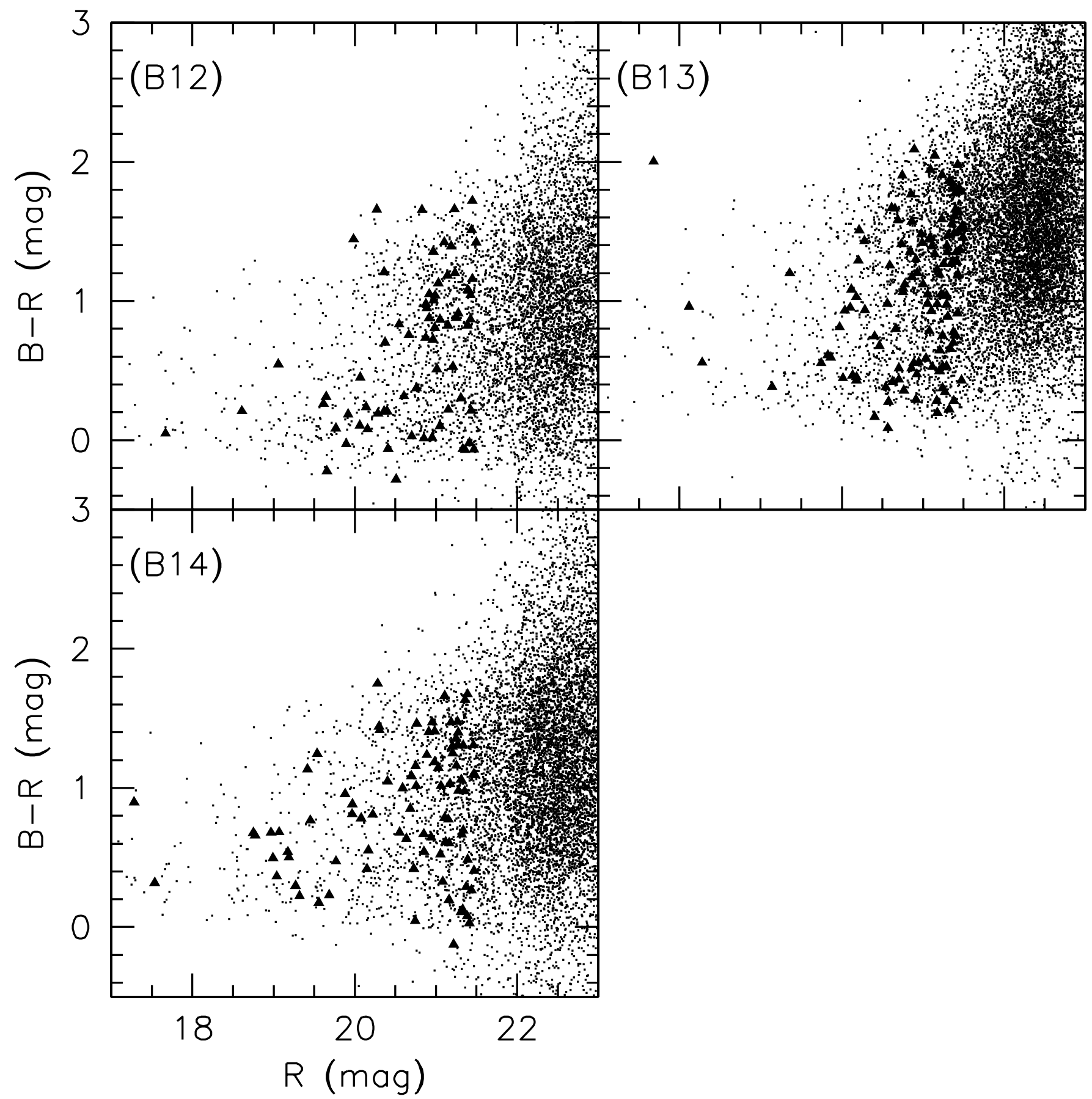

Fig. A.15. Shown are color-magnitude diagrams for our candidates B7, B8, B9 and B11. For an explanation of the plot see the caption from Fig. A.13. 